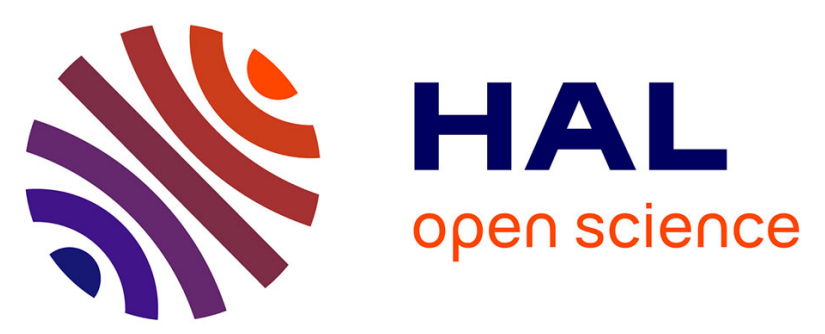

\title{
Highly resolved large eddy simulations of a binary mixture flow in a cavity with two vents: Influence of the computational domain.
}

Elie Saikali, Gilles Bernard-Michel, Anne Sergent, Christian Tenaud, R. Salem

\section{- To cite this version:}

Elie Saikali, Gilles Bernard-Michel, Anne Sergent, Christian Tenaud, R. Salem. Highly resolved large eddy simulations of a binary mixture flow in a cavity with two vents: Influence of the computational domain.. International Journal of Hydrogen Energy, 2019, 44 (17), pp.8856-8873. 10.1016/j.ijhydene.2018.08.108 . hal-02086328

\section{HAL Id: hal-02086328 https://hal.science/hal-02086328}

Submitted on 1 Apr 2019

HAL is a multi-disciplinary open access archive for the deposit and dissemination of scientific research documents, whether they are published or not. The documents may come from teaching and research institutions in France or abroad, or from public or private research centers.
L'archive ouverte pluridisciplinaire HAL, est destinée au dépôt et à la diffusion de documents scientifiques de niveau recherche, publiés ou non, émanant des établissements d'enseignement et de recherche français ou étrangers, des laboratoires publics ou privés. 


\title{
Highly resolved large eddy simulations of a binary mixture flow in a cavity with two vents: influence of the computational domain.
}

\author{
E. Saikali ${ }^{1,2}$, G. Bernard-Michel ${ }^{1}$, A. Sergent ${ }^{2,3}$, C. Tenaud ${ }^{2}$ and R. Salem ${ }^{1}$ \\ ${ }^{1}$ CEA Saclay (DEN-DANS-DM2S-STMF-LIEFT), F-91191 Gif-Sur-Yvette cedex, France, \\ elie.saikali@cea.fr, gilles.bernard-michel@cea.fr \\ ${ }^{2}$ LIMSI-CNRS, Université Paris-Saclay, F-91405 Orsay, France \\ elie.saikali@limsi.fr, anne.sergent@limsi.fr, christian.tenaud@limsi.fr \\ ${ }^{3}$ Sorbonne Université, UFR 919 Ingénierie, F-75005, Paris, France
}

\begin{abstract}
In this article, numerical results from a highly resolved large eddy simulations (LES) of an airhelium buoyant jet developing in a two vented cavity are presented. The simulated configuration mimics the helium-release experiment carried out at CEA Saclay in the framework of security assessment of indoor used hydrogen-based systems. The height of the enclosure was chosen so that a laminar-turbulent transition occurs approximately at the middle of the upstream direction. An exterior region, of different spatial dimensions, has been modelled in the computational domain in order to move the boundary conditions away from the vent surface and to approach the natural inlet/outlet conditions. A sensitivity analysis regarding the size of the exterior region is presented to define the minimum horizontal extension so that the flow inside the cavity is not furthermore influenced by bigger computational domains. We observe mainly that applying an ambient equilibrium-hydrostatic pressure outlet condition directly on the surface of the vent reduces the volume of the air inflow, and thus predicts larger helium mass inside the cavity, in contrary with the cases where an exterior region is considered. A qualification analysis shows that the sub-grid scale model plays a small role in the calculations and thus implies that the LES predictions approach the direct numerical simulation (DNS) solution. Analysis carried out on the time-averaged helium field depict a stratified regime not situated in the framework of the theoretical model used in safety pre-calculations.
\end{abstract}

Keywords: Vented cavity, large eddy simulations, buoyant jet, air-helium mixture, stratification, hydrogen safety.

\section{Introduction}

Systems using hydrogen as an energy carrier will extensively appear in our every day life over various range of scales; say transport and energy conversion applications for instance. It is necessary to place the hydrogen system in confined environments to isolate them from the human (user or public) and from material and environmental weather conditions. A typical accidental situation is when hydrogen escapes with a moderate flow-rate inside the environment. In such scenario, a hydrogen-air plume/jet rises and leads to the formation of a highly flammable and dangerous mixture with hazardous consequences due to the concentration accumulation/stratification. In this framework, configurations with passive ventilation are often 
considered to help in reducing the concentration accumulation and thus mitigate the risk induced in such scenarios. A simplified configuration is used in this work to analyse and predict the concentration distribution in the environment resulting from an accidental leakage. The problem is described as a hydrogen release (injection) inside a two vented cavitiy.

In the present paper, we simulate by large eddy simulations (LES) a particular flow regime described with a dimensionless Richardson number (Ri) at the release position slightly less than unity [1] . In such cases, a buoyant jet is predicted to develop inside the medium where a transition from a jet (dominant by inertial forces) into a plume (dominant by buoyancy forces) takes place upstream in a short distance above the release position [2]. The complete three dimensional (3D) cartesian geometry is considered without a priori made of any axi-symmetrical assumptions.

A particle image velocimetry (PIV) experiment has been carried out by Bernard-Michel et al. [3] at CEA Saclay in parallel with the numerical simulations to provide some information regarding the velocity distribution in two dimension vertical slices. LES-PIV comparisons in 2D plane sections can be reviewed from [4]. Taking into account the significance of the density ratio and since Bernard-Michel and Houssin show in [5] that helium can be considered as a good substitute for hydrogen in moderate release situations, helium injection is considered in the experimental and numerical works.

From a physical point of view, correctly mimicking the physics of the release problem is a challenging numerical task, especially capturing laminar-to-turbulent transition that is well known to occur within a short distance above the release position, the wide range of time and length scales, the sharp density gradients and thus instabilities [6]. Numerically speaking, specifying the natural boundary conditions at the inlet/outlet surfaces in semi-confined cavities is not an easy task and remains an open problem. This issue is rather more difficult when two vented cavities are considered since a vent can have an opposite flow orientation along its surface at the same time [7].

In the present paper, we aim at presenting a convergence study regarding the influence of modeling, in the numerical domain, an exterior region. Following such an approach, we can move the boundary conditions away from the vent surfaces from which we expect to better reproduce the flow pattern inside the cavity and basically the interaction between the fluid inside the cavity and the exterior environment.

A brief outline of the paper follows. The model and the numerical methods are discussed in section two. Section three is devoted to the convergence study carried out on the influence of the computational domain, in addition to the quality of the performed LES. In section four, the influence of the computational domain on the flow pattern is detailed. Section five reproduces the helium distribution in the cavity where a preliminary comparison versus the theoretical model of Linden et al. [8] is presented. Concluding remarks and future perspectives are finally stated in section six.

\section{Model, numerical setup and methods}

\subsection{Physical and experimental configurations}

The numerical configuration mimics the experimental study that has been carried out at CEA Saclay (LIEFT) to explore the behavior of an air-helium buoyant jet developing inside a two vented cavity. In order to facilitate the notation, we refer to the physical properties of the injected helium by the subscript ${ }_{\text {inj }}$ and those of the ambient air by the subscript ${ }_{\text {amb. }}$. The experimental 
set-up has been identified so that the desired flow regime, i.e. a turbulent buoyant jet, is expected to develop in the cavity $\left(\mathrm{Ri}_{\text {inj }}<1\right)$.

Through a cylindrical pipe, pure helium is injected into a parallelepiped cavity with a fixed volumetric flow-rate $Q=5.4581 . \mathrm{min}^{-1}$ (corresponding to $5 \mathrm{Nl} \cdot \mathrm{min}^{-1}$ of Helium). The cavity is subjected to a passive ventilation system with two vents and is initially filled with pure air at rest. Having a cross-sectional diameter $d=10^{-2} \mathrm{~m}$, a long enough injection pipe is used to ensure that a Poiseuille flow is well developed during the experiment. The dimensions of the cavity are the following: height $H=14.9 \times 10^{-2} \mathrm{~m}$, horizontal width $W=4.9 \times 10^{-2} \mathrm{~m}$ and span-wise length $L=5 \times 10^{-2} \mathrm{~m}$ as sketched in figure 1 . Moreover, the two vents are considered to be identical and both located on the right vertical wall, each having a surface area $5 \times 2.9 \times 10^{-4} \mathrm{~m}^{2}$. The solid boundaries of the cavity are formed of a plexi-glass with thickness $5 \times 10^{-3} \mathrm{~m}$.

The followed orientation of three dimensional (3D) direct cartesian labels is shown in figure 1 , where the origin of the coordinates is placed in the center of the top injection pipe. The $x$ direction is normal to the plane of the vents while the span-wise direction $(y)$ orients in the upstream direction parallel to the vents. The vertical $O z$ axis is situated along the height of the cavity, confounded with the axial axis of the cylindrical tube.
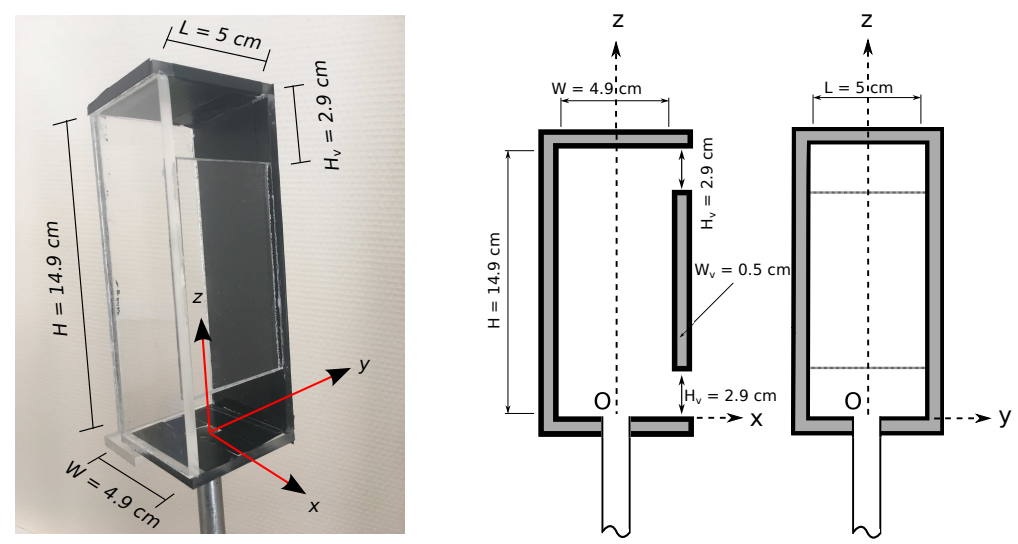

Figure 1: CEA Saclay (LIEFT) experimental configuration defined with the cartesian axis orientation. Left: 3D view, middle: 2D section perpendicular to the vents plane and containing the injection pipe, right: 2D section parallel to the vents surfaces exhibiting the two vents dimensions and containing the injection pipe.

The dimensions of the studied configuration are in accordance with the theoretical literature review of buoyant jets rising in an infinite medium. In particular, the height of the cavity $H$ is high enough so that a laminar-turbulent transitional flow can occur at $H / d \gg 5$ [1]. In addition, assuming that the buoyant jet spreading angle is in-between $11-12^{\circ}$ [9], the length $L$ and the width $W$ of the cavity are taken large enough to weaken the interaction of the jet boundaries with the side walls.

The temperature has been verified during the experiment to be constant (measured as $T=25^{\circ}$ C) and the isothermal assumption has been used. As far as the cavity is ventilated, the isobaric condition is assumed and the thermodynamic pressure $P_{\text {thm }}$ is taken constant and uniform; $P_{\text {thm }}=$ $10^{5} \mathrm{~Pa}$.

Following the isothermal and isobaric assumptions, the physical properties of the injected and ambient fluids are stated in table 1 . The density variation in the considered configuration is clearly noted where the injected fluid is approximately 7 times lighter than the ambient air. 


\begin{tabular}{cccc}
\hline Fluid & $\begin{array}{c}\text { Density } \\
{\left[\mathrm{kg} \cdot \mathrm{m}^{-3}\right]}\end{array}$ & $\begin{array}{c}\text { Dynamic viscosity } \\
{\left[\times 10^{-5} \mathrm{~kg} \cdot \mathrm{m}^{-1} \cdot \mathrm{s}^{-1}\right]}\end{array}$ & $\begin{array}{c}\text { Molar mass } \\
{\left[\times 10^{-2} \mathrm{~kg} \cdot \mathrm{mol}^{-1}\right]}\end{array}$ \\
\hline \hline Injected (species 1) & $\rho_{\mathrm{inj}}=0.16148$ & $\mu_{\mathrm{inj}}=1.918$ & $M_{\mathrm{inj}}=0.4003$ \\
Ambient (species 2) & $\rho_{\mathrm{amb}}=1.16864$ & $\mu_{\mathrm{amb}}=1.792$ & $M_{\mathrm{amb}}=2.897$ \\
\hline \hline
\end{tabular}

Table 1: Working fluids: physical properties at $T=25^{\circ} \mathrm{C}$ and $P_{\mathrm{thm}}=10^{5} \mathrm{~Pa}$.

However, the variation of the dynamic viscosity is rather comparable. At the injection, the dimensionless Richardson and Reynolds numbers are respectively $\operatorname{Ri}_{\text {inj }}=g\left(\rho_{\text {amb }}-\rho_{\text {inj }}\right) d /\left(\rho_{\text {inj }} u_{\text {inj }}^{2}\right) \approx$ $0.114<1$ and $\operatorname{Re}_{\text {inj }}=\rho_{\text {inj }} u_{\text {inj }} d / \mu_{\text {inj }} \approx 195$, where $u_{\text {inj }}$ is the maximum velocity, $u_{\text {inj }} \approx 2.3 \mathrm{~m} . \mathrm{s}^{-1}$.

\subsection{LES Governing equations}

The flow conservation equations are considered; mainly for the mass, momentum and species. The equation of state for a binary gas mixture formulation is used to relate the mixture's density with the species mass fractions [10]. It is not necessary to solve furthermore for an additional energy equation as far as the isothermal and isobar conditions are valid.

As far as the dimensionless Mach number is small $\left(\mathrm{Ma}=7 \times 10^{-3}<0.1\right)$ and since we consider a significant ambient-to-injection density ratio $\rho_{\mathrm{amb}} / \rho_{\text {inj }} \approx 7.24$, the considered flow problem is thus oriented towards simulating a mass variation rather than capturing and solving for the acoustic waves [11]. Therefore, the low Mach number (LMN) approximation is valid in this study where all acoustic waves are filtered out. Following an asymptotic analysis, the pressure of the fluid splits into the sum of a spatially uniform thermodynamic pressure $p$ and a hydrodynamic pressure $P(\mathbf{x}, t)$ dependent of both space and time [12]. Here, $\mathbf{x}$ denotes the space coordinate vector and $t$ the time .

The LES system of equations is obtained by applying a spatial filter (denoted by ${ }^{-}$) over the set of governing equations and using density weighted Favre quantities (denoted for a considered quantity $\varphi$ as $\widetilde{\varphi}=\overline{\rho \varphi} / \bar{\rho}$ ). It is expressed as

$$
\begin{aligned}
& \frac{\partial \bar{\rho}}{\partial t}+\frac{\partial}{\partial x_{i}}\left(\bar{\rho} \widetilde{u}_{i}\right)=0 \\
& \frac{\partial \bar{\rho} \widetilde{u}_{j}}{\partial t}+\frac{\partial}{\partial x_{i}}\left(\bar{\rho} \widetilde{u}_{j} \widetilde{u}_{i}\right)=-\frac{\partial \bar{P}}{\partial x_{j}}+\frac{\partial \bar{\tau}_{i j}}{\partial x_{i}}-\frac{\partial \bar{\tau}_{i j}^{\mathrm{SGS}}}{\partial x_{i}}+\bar{\rho} g_{j} \\
& \frac{\partial \bar{\rho} \widetilde{Y}_{1}}{\partial t}+\frac{\partial}{\partial x_{i}}\left(\bar{\rho} \widetilde{u}_{i} \widetilde{Y}_{1}\right)=\frac{\partial}{\partial x_{i}}\left(D \bar{\rho} \frac{\partial \widetilde{Y}_{1}}{\partial x_{i}}\right)-\frac{\partial \bar{\xi}_{i}^{\mathrm{SGS}}}{\partial x_{i}} \\
& \bar{\rho}=\frac{p}{R T}\left(\frac{\widetilde{Y}_{1}}{M_{\mathrm{inj}}}+\frac{\widetilde{Y}_{2}}{M_{\mathrm{amb}}}\right)^{-1}
\end{aligned}
$$

where

$$
\bar{\tau}_{i j}-\frac{1}{3} \bar{\tau}_{k k} \delta_{i j}=2 \widetilde{\mu}\left(\bar{S}_{i j}-\frac{1}{3} \bar{S}_{k k} \delta_{i j}\right),
$$


with the symmetrical strain rate tensor: $\bar{S}_{i j}=\frac{1}{2}\left(\frac{\partial \widetilde{u}_{j}}{\partial x_{i}}+\frac{\partial \widetilde{u}_{i}}{\partial x_{j}}\right)$, and $\delta_{i j}$ the Kronecker symbol,

$$
\bar{\tau}_{i j}^{\mathrm{SGS}}=-\bar{\rho}\left(\widetilde{u}_{i} \widetilde{u}_{j}-\widetilde{u_{i} u_{j}}\right) \quad \text { and } \quad \bar{\xi}_{i}^{\mathrm{SGS}}=-\bar{\rho}\left(\widetilde{u}_{i} \widetilde{Y}_{1}-\widetilde{u_{i} Y_{1}}\right) \text {. }
$$

Here, the filtered mixture density is $\bar{\rho}$, while $\widetilde{Y}_{1}$ and $\widetilde{Y}_{2}$ denote respectively the helium (index 1) and air (index 2) mass fractions and satisfy the unity summation $\widetilde{Y}_{1}+\widetilde{Y}_{2}=1 . \widetilde{u}_{i}$ is the mass weighted component in the $i$-direction, of the velocity vector $\widetilde{\mathbf{u}}=\left(\widetilde{u}_{1}, \widetilde{u}_{2}, \widetilde{u}_{3}\right)$.

The mixture diffusion coefficient is considered to be uniform and constant in the present work; $D=6.91 \times 10^{-5} \mathrm{~m}^{2} \cdot \mathrm{s}^{-1} \cdot R=8.314 \mathrm{~J} \cdot \mathrm{K}^{-1} \cdot \mathrm{mol}^{-1}$ denotes the classical specific gas constant. $\bar{\tau}_{i j}$ is the viscous stress tensor considered for a Newtonian fluid here and $\mu$ denotes the dynamic viscosity defined for a binary gas mixture as in [13]. We denote by $g_{j}=(0,0,-g)$ the acceleration vector of the gravity.

$\bar{\tau}_{i j}^{\mathrm{SGS}}$ and $\bar{\xi}_{i}^{\mathrm{SGS}}$ are respectively the sub-grid scale (SGS) Reynolds stress tensor and the subgrid scale scalar flux vector that need to be modeled for closing the LES system of equations. The classical Smagorinsky model [14] that is based on an eddy-viscosity assumption, is employed to express the components of the SGS $\bar{\tau}_{i j}^{\mathrm{SGS}}$ tensor as follows:

$$
\begin{gathered}
\bar{\tau}_{i j}^{\mathrm{SGS}}-\frac{1}{3} \bar{\tau}_{k k}^{\mathrm{SGS}} \delta_{i j}=2 \mu_{\mathrm{SGS}}\left(\bar{S}_{i j}-\frac{1}{3} \bar{S}_{k k} \delta_{i j}\right), \\
\mu_{\mathrm{SGS}}=\bar{\rho}\left(C_{s} \Delta\right)^{2} \sqrt{2 \bar{S}_{i j} \bar{S}_{i j} .}
\end{gathered}
$$

Here, $\Delta$ is the filter width taken to be equal to the cell volume $\left(\delta_{x} \delta_{y} \delta_{z}\right)^{1 / 3}$, with $\delta_{x}, \delta_{y}$ and $\delta_{z}$ are respectively the effective mesh spacing per direction. The Smagorinsky coefficient is considered in this work constant and uniform; $C_{s}=0.18$.

The unresolved sub-grid scale scalar flux vector $\bar{\xi}_{i}^{\mathrm{SGS}}$ is modeled by using a classical Fourier's law as:

$$
\bar{\xi}_{i}^{\mathrm{SGS}}=-\frac{\mu_{\mathrm{SGS}}}{S c_{\mathrm{SGS}}} \frac{\partial \widetilde{Y}_{1}}{\partial x_{i}}
$$

where the SGS Schmidt number is assumed in the whole domain constant and uniform; $S c_{\mathrm{SGS}}=$ 0.7 (to compare with $\mathrm{Sc}_{\mathrm{inj}}=\mu_{\text {inj }} /\left(D \rho_{\mathrm{inj}}\right) \approx 1.719$ and $\left.\mathrm{Sc}_{\mathrm{amb}} \approx 0.222\right)$. For simplicity, we remove all filter symbols in what follows.

\subsection{Numerical set-up and computational domains}

The numerical study is performed on exactly the same configuration as the one used in the CEA experiments. Th height $(h)$ of the injection pipe must be selected so as to ensure that it is long enough to obtain a well developed flow independently of the prescribed velocity profile at the bottom inlet of this pipe. A height of $10 \times 10^{-2} \mathrm{~m}$, has been employed. Results show (fig. 2) that a unique value of the vertical velocity is reached at $8 \mathrm{~cm}$ above the bottom inlet, whatever a flat or a parabolic profile is initially prescribed at the bottom of the pipe.

We perform numerical computations on six different configurations labelled from $0_{x}, 1_{x}$ to $5_{x}$. In configuration $0_{x}$, the flow is simulated only inside the pipe and the cavity. A description of the computational domain $0_{x}$ can be seen in figure 3 with two vertical planes perpendicular and parallel to the vents, and exhibiting the pipe and the two vents dimensions. In this case, the 


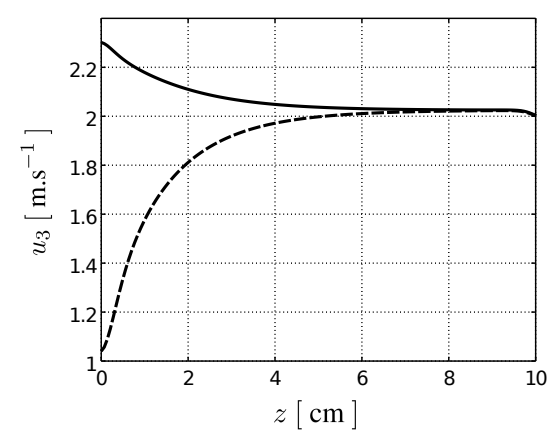

Figure 2: Vertical velocity distribution along the vertical axis of the injection pipe. Solid line denotes the profile established with a Poiseuille velocity profile prescribed at the bottom inlet. Dashed line denotes the profile obtained with a prescribed flat velocity profile.
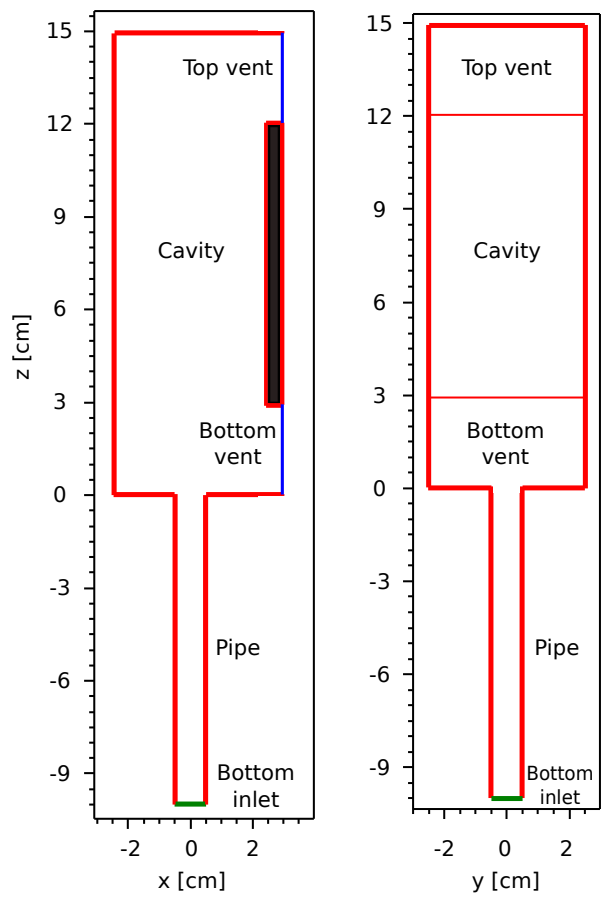

Figure 3: The numerical computational domain $0_{x}$ described by two vertical planes perpendicular (left) and paralle (right) to the vents, and exhibiting the pipe and the two vents dimensions. The black shaded region denote a non-fluid zone reproducing the thickness of the plexi-glass wall.

outlet boundary conditions are applied just at the exterior vent surfaces to reproduce exchanges between the cavity and the exterior environment.

For the remaining five configurations $\left(1_{x}\right.$ to $5_{x}$ ), we consider an exterior domain with different dimensions to be attached directly with the vent surfaces. This methodology helps in moving the outlet boundary conditions a bit away from the cavity. A solid layer with a width of $5 \times 10^{-3} \mathrm{~m}$ is modeled around the vents to reproduce the plexi-glass used in the experiment. The horizontal 
extension of the exterior region is $L x$, while the span-wise length is $L+(2 \times L y)$ and the height is $H+(2 \times L z)$. In the present work, we aim at performing a convergence study on the size of the exterior region by only varying $L x$ from $2 \mathrm{~cm}$ to 10.125 , in a geometrical sequence of ratio $q=1.5$. Extensions in the span-wise and vertical directions are kept constant throughout the work; $L y=L z=2 \mathrm{~cm}$. A description of the computational domain is depicted in figure 4 with two vertical planes perpendicular and parallel to the vents, and exhibiting the pipe, exterior region and the two vents dimensions.
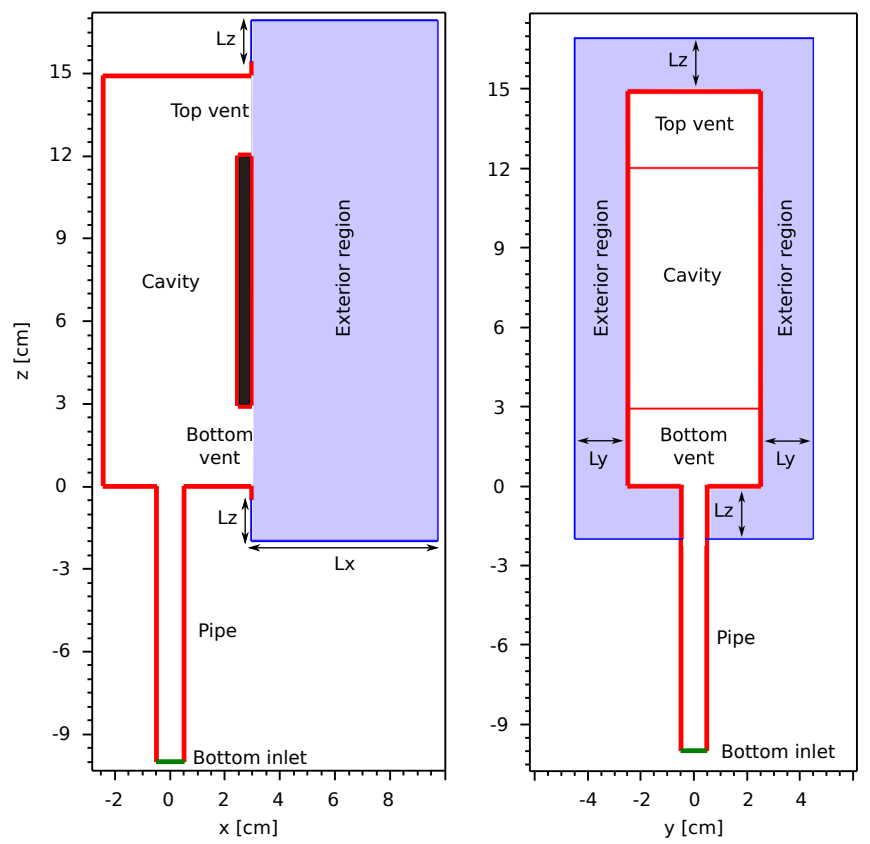

Figure 4: The numerical computational domains employed for configurations $1_{x}$ to $5_{x}$ described by two vertical planes perpendicular (left) and parallel (right) to the vents, and exhibiting the pipe, exterior domain and the two vents dimensions. The black shaded region denote a non-fluid zone reproducing the thickness of the plexi-glass wall.

The complete numerical description of the six computational domains is presented in table 2 .

\begin{tabular}{c|c|cc}
\hline \hline Configuration & $L x[\mathrm{~cm}]$ & Cell numbers & MPI procs \\
\hline \hline $\mathbf{0}_{x}$ & & $1,134,404$ & 20 \\
$\mathbf{1}_{x}$ & 2 & $2,129,220$ & 40 \\
$\mathbf{2}_{x}$ & 3 & $2,609,476$ & 40 \\
$\mathbf{3}_{x}$ & 4.5 & $3,329,860$ & 60 \\
$\mathbf{4}_{x}$ & 6.75 & $4,427,588$ & 80 \\
$\mathbf{5}_{x}$ & 10.125 & $6,108,484$ & 100 \\
\hline \hline
\end{tabular}

Table 2: Description of the 3D numerical configurations. 


\subsection{CFD code and numerical methods}

Numerical simulations are carried out by using the open source CFD code "TRio_U Software for Thermohydraulics" (CEA TRUST-TrioCFD) [15]. At each time iteration, the LES system of equations is treated in a sequential way by employing a semi-implicit scheme, with an implicit treatment for the diffusion-viscous terms while explicitly the convective ones [16, 17]. To ensure the numerical stability of the scheme, the time step $\delta t$ employed at each iteration is selected to satisfy the convective Courant-Friedrichs-Lewy condition $\left(\mathrm{CFL}_{\text {conv }}\right)$;

$$
\delta t=\delta t_{\mathrm{conv}}=\min \left(\frac{V_{\mathrm{cell}}}{\zeta_{\mathrm{cell}}}\right),
$$

where $\zeta_{\text {cell }}\left[\mathrm{m}^{3} \cdot \mathrm{s}^{-1}\right]$ corresponds to the volumetric flux entering at each arbitrary control volume (cell) of volume $V_{\text {cell }}$.

The discretization is carried out on a staggered grid by employing a Finite Difference Volume (FDV) method; the discretization of each term is performed by integrating over a control volume where the diffusion gradient terms are approximated by a linear difference equation. A second order Rational Range-Kutta (RRK2) scheme [18] is used for the time integration. A second ordered centered scheme is used to discretize all spatial-derivative terms except for the species convective term appearing in equation (3) where a quadratic upstream interpolation for convective kinematics (QUICK) scheme of third order is employed to ensure both, the monotonicity property and that $Y_{1} \in[0,1]$ (boundedness) [19]. The conjugate gradient method (CGM) [20] is used to iterate the linear systems which result from the implicit treatment of the viscous-diffusive terms.

To deal with the pressure-velocity coupling problem, an incremental projection method is employed [21]. In the CEA TRUST-TrioCFD code, the Poisson equation is written with a constant coefficient formulation where the pressure increment $\phi=P^{t+\delta t}-P^{t}$ is the tracked variable. An iterative symmetric successive over relaxation (SSOR) method is used to solve for $\phi$. Finally, the velocity field is updated to ensure the mass balance presented in equation (1).

\subsection{Prescribed initial and boundary conditions}

At the initial state, the cavity is totally filled with pure air $\left(Y_{1}=0\right)$ beeing at rest $(\mathbf{u}=0)$ while the injection pipe is initialized with a smooth vertical profile of helium mass fraction, decreases from $Y_{1}=1$ (pure helium) at the bottom of the pipe, where the inlet boundary condition is prescribed, to $Y_{1}=0$ (pure air) approaching the top of the pipe with a vanishing vertical gradient of the mass fraction. The smooth distribution is considered to initially avoid mass fraction diffusion and to reduce the stiff concentration gradients at the first iterations of the simulation.

The boundaries of the complete domain $\Omega$ can be classified into three distinct types: solid wall boundaries $\left(\partial \Omega_{w}\right)$ reproducing the plexi-glass in the real experiment, inlet boundary $\left(\partial \Omega_{i n}\right)$ representing the surface where pure helium is injected and the outlet boundaries $\left(\partial \Omega_{\text {out }}\right)$ where the exchange between the interior and exterior environments is modeled. We particularly consider the following decomposition

$$
\partial \Omega=\partial \Omega_{w} \cup \partial \Omega_{\text {in }} \cup \partial \Omega_{\text {out }} .
$$

The position of the three boundaries are depicted in figure 5 from a 3D view of the computational domains. Red surfaces indicate the position of the wall boundaries $\partial \Omega_{w}$, the injection surface $\partial \Omega_{\text {in }}$ is colored in yellow and finally the blue surfaces show the position of the outlet 
surfaces $\partial \Omega_{\text {out }}$. The pipe is identical in the six configurations and is sketched in subfigure (a). Subfigure (b) represents the 3D computational domain $0_{x}$ where outlet conditions are prescribed just at the external surface of the vents, while subfigure (c) depicts the configurations $1_{x}$ to $5_{x}$ where the shape of the external domain could be clearly recognized.

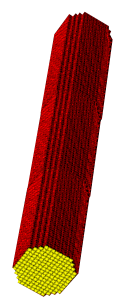

(a)

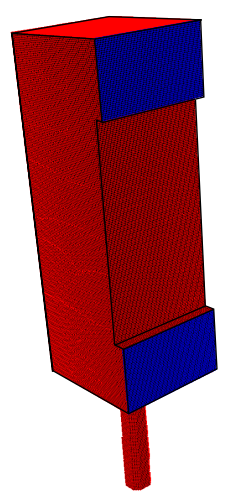

(b)

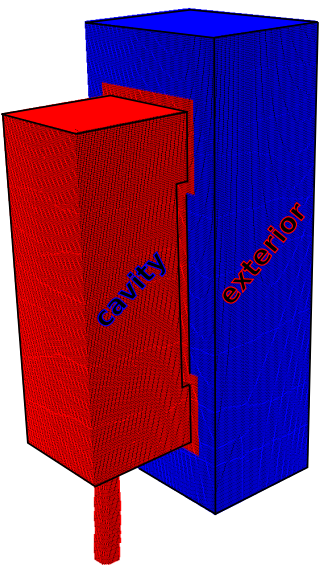

(c)

Figure 5: 3D view of the simulated configurations showing the positions of the prescribed boundary conditions: red surfaces denoting the wall boundaries, yellow surface represents the inlet boundary, while blue surfaces describes the position of the numerical outlet boundaries. (a): the modeled pipe in the six cases, (b): configuration $0_{x}$ and (c) : configurations $1_{x}$ to $5_{x}$.

The implemented boundary conditions are the following. On $\partial \Omega_{w}$, we prescribe a no-slip boundary condition for $\mathbf{u}$ with a zero-gradient homogeneous Neumann condition for all the scalars $\rho, Y_{1}$ and $P$. At $\partial \Omega_{i n}$, a fixed convective mass flux equal to $\rho_{\text {inj }} Q_{v}$ is imposed with a flat profile for $\rho=\rho_{\text {inj }}$ and $Y_{1}=1$, where $Q_{v}$ denotes the injection volumetric flow-rate. A parabolic profile is imposed for $\mathbf{u}$ so that the velocity vector is oriented along the vertical $z$-direction with a maximum velocity $u_{3}=2.33 \mathrm{~m} \cdot \mathrm{s}^{-1}$, corresponding to a mass flow rate of $5 \mathrm{NL} \cdot \mathrm{min}^{-1}$. The vertical velocity profile at $\partial \Omega_{\text {in }}$ is thus prescribed as:

$$
u_{3}=\epsilon_{Q} \times\left[d^{2} / 4-\left(x^{2}+y^{2}\right)\right],
$$

where $\epsilon_{Q}$ is a correction parameter used to ensure the volumetric flow-rate conservation and thus calculated as

$$
\epsilon_{Q}=\frac{Q_{v}}{\iint_{\partial \Omega_{i}} u_{3_{*}} d x d y} .
$$

At $\partial \Omega_{\text {out }}$, an ambient-equilibrium pressure $P=-\rho_{\text {amb }} g z$ is imposed at a considered height $z$, coupled with a homogeneous Neumann condition for $\mathbf{u}$. Regarding the remaining fluid variables, a flow orientation test is carried out. Let us denote by $\hat{\eta}$ is the outward unit normal at the outlet surfaces. If $\mathbf{u} \cdot \hat{\eta} \geq 0$, we apply a homogeneous Neumann condition on $\rho$ and $Y_{1}$. Otherwise, an inflow takes place at the vent and a Dirichlet condition is imposed; $\rho=\rho_{\text {amb }}$ and $Y_{1}=0$. Let us recall that the physical properties of helium and air at $T=25^{\circ} \mathrm{C}$ and $P_{\mathrm{thm}}=10^{5} \mathrm{~Pa}$ can be found in table 1 . 


\subsection{Choice of the mesh}

Similar to the procedure described in [16], a grid convergence study has been carried out to identify the required mesh size that can reproduce convergent LES predictions. In this study, only configuration $0_{x}$ is used and seven different uniform meshes are generated where the cell step size decreases from $2 \times 10^{-3}$ to $4.14 \times 10^{-4} \mathrm{~m}$, with a geometric factor $r=1.3$.

Both time-averaged fields, respectively those of $Y_{1}$ and the velocity magnitude $|\mathbf{u}|=\left(u_{1}^{2}+u_{2}^{2}+\right.$ $\left.u_{3}^{2}\right)^{1 / 2}$, have shown satisfactory converged results as far as the grid step size is less than $7 \times 10^{-4}$ $\mathrm{m}$. While the convergence of the rms values is much slower, it can be stated that satisfactory converged $r m s$ values have also been reached.

In the followings, a uniform unstructured cubic mesh of size $\delta=7 \times 10^{-4} \mathrm{~m}$, per cell in each direction, has then been generated for the six configurations. Following the work of Chhabra $e t$ al. [22], the Kolmogorov length scale can be estimated at $\eta=2.1 \times 10^{-4} \mathrm{~m}$ (leading to a ratio $\delta / \eta=3.3)$.

\subsection{Quasi-steadiness and statistical recordings}

Six simulations covering 110 seconds of physical time have been performed. We assume that a quasi-steady state is reached after $t=70 \mathrm{~s}$ and thus statistical fields have been calculated over a unique window ([70,110] seconds) with an accumulation every time step. Defining the height of the cavity as a reference length $\left(H_{r}=14.9 \mathrm{~cm}\right)$ and the maximum injection velocity as a reference velocity $\left(\mathbf{u}_{r}=2.33 \mathrm{~m} . \mathrm{s}^{-1}\right)$, the $40 \mathrm{~s}$ of statistical accumulation corresponds to 625 of non-dimensional time units.

We denote respectively by $\langle\cdot\rangle_{t}$ and $\operatorname{rms}\{\cdot\}_{t}$ the time averaged and the root mean square (rms) operators. For a considered quantity $\varphi(\mathbf{x}, t)$, the fields are thus calculated respectively as

$$
\begin{gathered}
<\varphi(\mathbf{x}, t)>_{t}=\frac{1}{40} \int_{70}^{110} \varphi(\mathbf{x}, t) d t, \\
\operatorname{rms}\{\varphi(\mathbf{x}, t)\}_{t}=\left(\frac{1}{40} \int_{70}^{110}\left(\varphi^{\prime}(\mathbf{x}, t)\right)^{2} d t\right)^{1 / 2} .
\end{gathered}
$$

Here, $\varphi^{\prime}$ denotes the fluctuating part of $\varphi$ centered on the mean value, defined as:

$$
\varphi^{\prime}(\mathbf{x}, t)=\varphi(\mathbf{x}, t)-<\varphi(\mathbf{x}, t)>_{t} .
$$

\subsection{Statistical accuracy}

In order to check the accuracy of the performed statistics, histories of $Y_{1}$ and $|\mathbf{u}|$ at 14,161 fixed probes ( $17 \times 17 \times 49)$, uniformly distributed per direction inside the cavity, are recorded for each simulation with a frequency of $10^{3} \mathrm{~Hz}$. The numerical-statistical error (standard error of the mean) is defined as:

$$
\operatorname{Err}(\varphi)=\frac{\operatorname{rms}\{\varphi(t)\}_{t}}{\sqrt{\mathcal{N}}}
$$

where $\mathcal{N}$ denotes the number of independent samples and $\varphi(t)=\left\{Y_{1},|\mathbf{u}|\right\}$. The number of independent samples are deduced from the temporal evolution of the auto-correlation function (ACF) over a time lag $\tau$;

$$
r(\tau)_{\varphi(t)}=\frac{<\varphi^{\prime}(t) \cdot \varphi^{\prime}(t+\tau)>_{t}}{<\varphi^{\prime}(t)^{2}>_{t}}
$$


In particular, the uncorrelated data corresponds to the Taylor micro-scale $\lambda_{f}$ [23], defined as the intersection of a parabola fitting the distribution at the origin, with the time lag axis.

Using the recorded frequency, 40 seconds of statistical recordings correspond to $40 \times 10^{3}$ samples, which contain correlated and uncorrelated data. In configuration $4_{x}$ for example, it has been figured out that each consecutive 50 points on the profile of $Y_{1}$ are dependent while the number of correlated samples is 25 for $|\mathbf{u}|$. From equation (15), it can then be stated that $\operatorname{Err}\left(Y_{1}\right)=0.02 \%$ and $\operatorname{Err}(|\mathbf{u}|)=4.2 \times 10^{-3} \mathrm{~m} \cdot \mathrm{s}^{-1}$

\subsection{Computational cost}

The six simulations have been carried out on the CEA Saclay local cluster (Intel Xeon, E5$2680 \mathrm{~V} 2,2.8 \mathrm{GHz}, 128$ Go memory per node and infiniband QDR $40 \mathrm{Gbit}^{-1}{ }^{-1}$ ). The total wall time computational cost is summarized in table 3.

\begin{tabular}{ccc}
\hline \hline Configuration & MPI procs & Time [hours] \\
\hline \hline $\mathbf{0}_{x}$ & 20 & 353.2 \\
$\mathbf{1}_{x}$ & 40 & 350 \\
$\mathbf{2}_{x}$ & 40 & 476.2 \\
$\mathbf{3}_{x}$ & 60 & 505.3 \\
$\mathbf{4}_{x}$ & 80 & 415.8 \\
$\mathbf{5}_{x}$ & 100 & 485.14 \\
\hline \hline
\end{tabular}

Table 3: Computational cost: simulations LES with a physical time $t=110$ seconds, CEA-Saclay CALLISTO cluster.

\section{Results: global numerical comparisons, convergence and validations}

\subsection{Global quantitative comparison: helium mass and flow-rates}

In order to perform a global quantitative comparison on the influence of the computational domain, we calculate the time-averaged total mass of helium $\left\langle\mathcal{M}_{\mathrm{He}}\right\rangle_{t}$ inside the cavity, helium mass flow-rate leaving the top vent $\left\langle q_{H e}^{\text {top }}\right\rangle_{t}$, volumetric flow-rate of air entering the bottom vent $<Q_{v}^{b o t}>_{t}$ and that of the mixture leaving from the top $<Q_{v}^{t o p}>_{t} ; \mathcal{M}_{H e}=\int_{V} \rho_{\text {inj }} X_{1} d V$, $q_{H e}^{i}=\int_{\partial \Omega_{\text {out }}^{i}} \rho_{\text {inj }} X_{1} u_{1} d \sigma$ and $Q_{v}^{i}=\int_{\partial \Omega_{\text {out }}^{i}} u_{1} d \sigma$ where $V$ is the volume of the cavity, $i=\{b o t$, top $\}$ and $\partial \Omega_{\text {out }}^{\text {bot }}, \partial \Omega_{\text {out }}^{\text {top }}$ are respectively the surface areas of the bottom and the top vents. The values are summarized in table 4 .

We can clearly note that the configuration $0_{x}$ overestimates the mass of helium $\left\langle\mathcal{M}_{H e}\right\rangle_{t}$ inside the cavity, compared to the remaining configurations (red boxes in figure 6). This is justified by the values of the volumetric flow-rates entering and leaving the cavity, respectively $<Q_{v}^{b o t}>_{t}$ and $<Q_{v}^{t o p}>_{t}$, which are lower in configuration $0_{x}$ (black circles in figure 6).

Regarding the configurations that employ an exterior region, we note that the total mass of helium is at least about $6.4 \%$ lower than that in $0_{x}$. Almost convergent values are predicted in the last two $4_{x}$ and $5_{x}$ configurations (values in the shaded region of figure 6 ). For instance, selecting the helium mass obtained in configuration $5_{x}$ as a reference value, the relative error of $\left\langle\mathcal{M}_{\mathrm{He}}\right\rangle_{t}$ estimated in configuration $4_{x}$ is arround $0.3 \%$. 


\begin{tabular}{c|cc|cc}
\hline \hline Configuration & $\begin{array}{c}\left\langle\mathcal{M}_{H e}\right\rangle_{t} \\
{\left[\times 10^{-6} \mathrm{~kg}\right]}\end{array}$ & $\begin{array}{c}\left\langle q_{H e}^{\text {top }}\right\rangle_{t} \\
{\left[\times 10^{-5} \mathrm{~kg} \cdot \mathrm{s}^{-1}\right]}\end{array}$ & $\begin{array}{c}\left\langle Q_{v}^{\text {bot }}\right\rangle_{t} \\
{\left[\times 10^{-4} \mathrm{~m}^{3} \cdot \mathrm{s}^{-1}\right]}\end{array}$ & $\begin{array}{c}\left\langle Q_{v}^{\text {top }}\right\rangle_{t} \\
{\left[\times 10^{-4} \mathrm{~m}^{3} \cdot \mathrm{s}^{-1}\right]}\end{array}$ \\
\hline \hline $\mathbf{0}_{x}$ & 9.02033 & 1.41644 & -2.57013 & 3.47496 \\
$\mathbf{1}_{x}$ & 8.10638 & 1.42059 & -2.81147 & 3.71449 \\
$\mathbf{2}_{x}$ & 8.30408 & 1.40970 & -2.80676 & 3.70839 \\
$\mathbf{3}_{x}$ & 8.20663 & 1.42378 & -2.77233 & 3.67504 \\
$\mathbf{4}_{x}$ & 8.47855 & 1.39376 & -2.60348 & 3.49892 \\
$\mathbf{5}_{x}$ & 8.45375 & 1.39996 & -2.60436 & 3.50027 \\
\hline
\end{tabular}

Table 4: Global time averaged quantities for configurations $0_{x}$ to $5_{x}$ : helium total mass, mass and volumetric flow-rates.

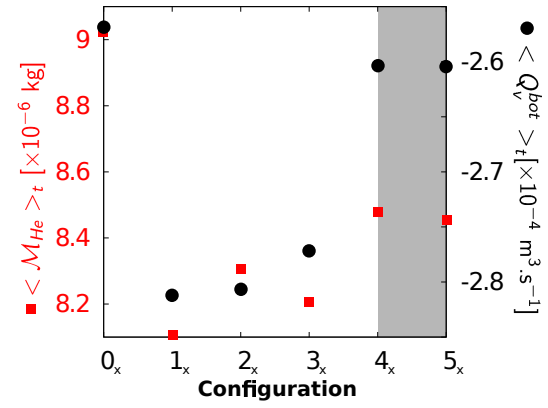

Figure 6: The evolution of the helium total mass $\left\langle\mathcal{M}_{H e}\right\rangle_{t}$ (red boxes) and the volumetric flow-rate crossing the bottom vent $\left\langle Q_{v}^{\text {bot }}\right\rangle_{t}$ (black circles) versus the computational domain.

\subsection{Convergence on the size of the exterior domain}

In this subsection, we present a quantitative convergence study on the size of the exterior domain based on the variation of $L x$. The mean quantities calculated at the same 14,161 probes described in subsection 2.8 are used. The fields predicted with configuration $5_{x}$ have been chosen as the reference values. The evolution of the relative error versus configurations $1_{x}$ to $4_{x}$ is plotted in figure 7 .
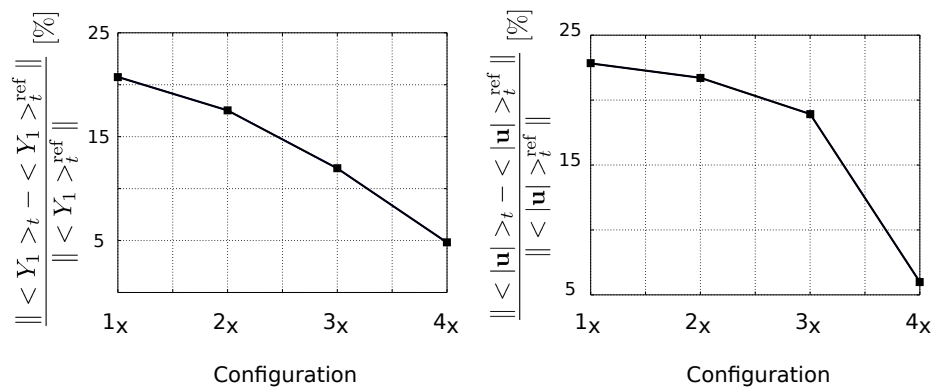

Figure 7: Evolution of the L2 norm mean relative error as a function of configurations $1_{x}$ to $4_{x}$. Left: helium mass fraction $Y_{1}$, right: velocity magnitude $|\mathbf{u}|$.

It can be stated that the results are satisfactory and we assume that a convergence is attained. 
Both graphs show that a significant decrease takes place where the mean mass fraction error decays from $21 \%$ to $4.8 \%$ and that of $|\mathbf{u}|$ from $23 \%$ to $6 \%$. We recall that with such an employed mesh size, the error on evaluating the mean of $Y_{1}$ and $|\mathbf{u}|$ is estimated to be respectively $8 \%$ and $12 \%$.

In conclusion, we can say that for a horizontal extension $L_{x} \geq 6.75 \mathrm{~cm}$ (configuration $4_{x}$ ), the interior cavity flow becomes almost independent on the discretized exterior region and no further influence will be recorded. Thus, unless stated otherwise, the results presented in the sequel for a simulation with an exterior domain corresponds to those obtained by configuration $4_{x}$.

\subsection{Numerical validations and LES qualification}

We have depicted previously that according to a carried grid convergence study, we could identify the cell size $\delta$ necessary for a good LES prediction. In this subsection, we look towards investigating whether the performed LES calculation is well solved or not. To illustrate, we consider the mid-vertical $x z$ plane situated at $y=0 \mathrm{~cm}$. An LES qualification similar to that carried out by Maragkos et al. while simulating a large helium buoyant plume in [24] is performed and the analysis is presented in what follows.

\subsubsection{Modeled to laminar viscosity ratio}

The ratio of the modeled SGS to the laminar mixture kinematic viscosity is calculated inside the cavity only (the modeled viscosity in the exterior domain is not taken into account). As presented in [25], this ratio serves to be a good indicator on the quality of the performed LES. Globally, we observe that at the quasi-steady state solution, $v_{\mathrm{SGS}} / v \leq 0.34$, confirming that a fine LES has been carried out here.

At the time $t=106$ seconds, the instantaneous modeled SGS viscosity $v_{\mathrm{SGS}}$, the laminar viscosity of the mixture $v$ and their corresponding ratio $v_{\mathrm{SGS}} / v$ are considered respectively in figure 8 . The iso-contours show that the highest values of $v_{\mathrm{SGS}}$ are recorded at the jet axis and in the recirculating zones located mainly in the top left corner and in the region between the jet-left wall (figure 8, left). This is confirmed as far as, by construction, the Smagorinsky's model is linearly dependent of the velocity gradients. The importance of the modeled $v_{\mathrm{SGS}}$ is reflected on the viscosity ratio (figure 8 right). It points on the high kinetic energy-dissipative regions.

\subsubsection{Kolmogorov length scale} tion

A Kolmogorov length scale $\eta_{\text {LES }}$ is estimated from the obtained LES results using the defini-

$$
\eta_{\mathrm{LES}}=\left(v^{3} / \epsilon_{\mathrm{LES}}\right)^{0.25}
$$

where the instantaneous total dissipation rate is evaluated as

$$
\epsilon_{\mathrm{LES}}=2\left(v_{\mathrm{SGS}}+v\right)\left(S_{i j} S_{j i}\right) .
$$

The instantaneous ( $t=106 \mathrm{~s}$ ) distribution of the Kolmogorov length scale $\eta_{\text {LES }}$ and the ratio of the grid spacing $\delta$ to $\eta_{\text {LES }}$ have been estimated again in the mid vertical $x z$-plane $(y=0)$. The iso-contour field is depicted in figure 9.

It is clear that $\eta_{\text {LES }}$ is dependent on the location and thus on the flow pattern, although the maximum ratio $\delta / \eta_{\text {LES }}$ is figured out to be 4 at the top ceiling and at most 3 elsewhere, which is relatively small in the practice of LES [24]. Assuming that the Smagorinsky model is correctly representing the unresolved scales, we can say that the LES is correctly representing the flow and that the LES prediction is not far from the DNS solution. 

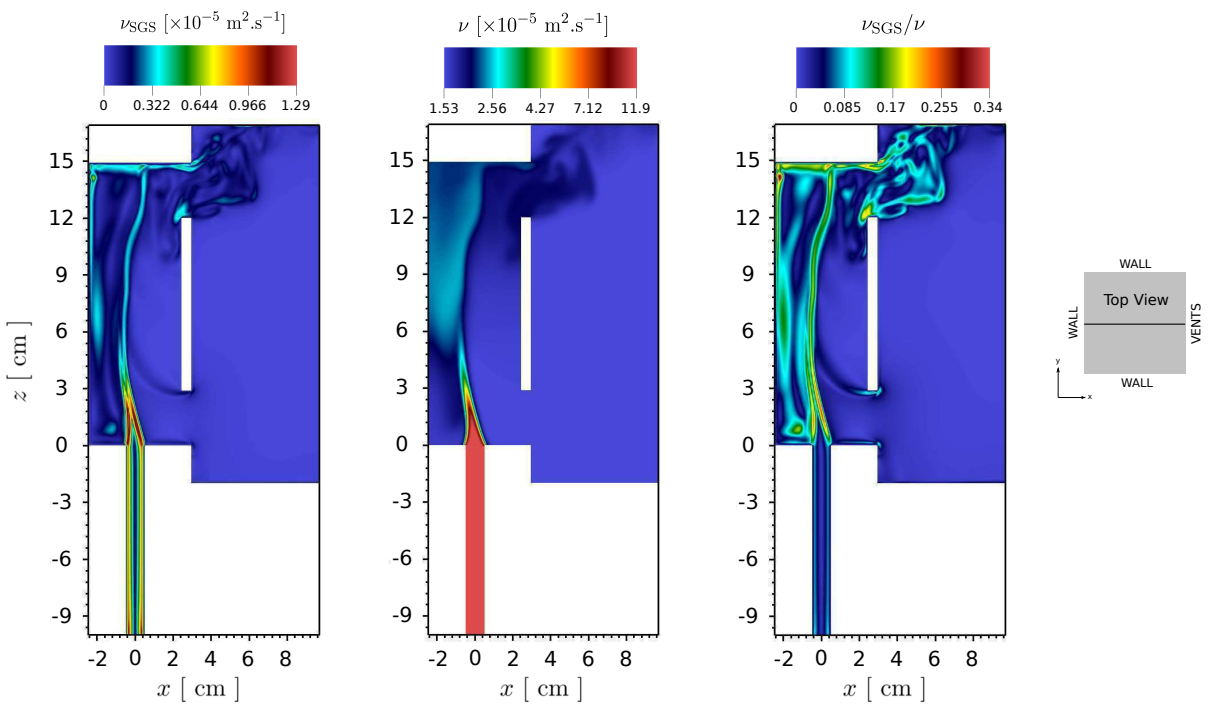

Figure 8: Instantaneous iso-contour plots at $t=106$ seconds in the mid-vertical $x z$-plane $(y=0 \mathrm{~cm})$. Left: SGS kinematic modeled viscosity $v_{\mathrm{SGS}}$, middle: mixture kinematic laminar viscosity $v$ (levels in a log scale), right: viscosity ratio $v_{\mathrm{SGS}} / v$.
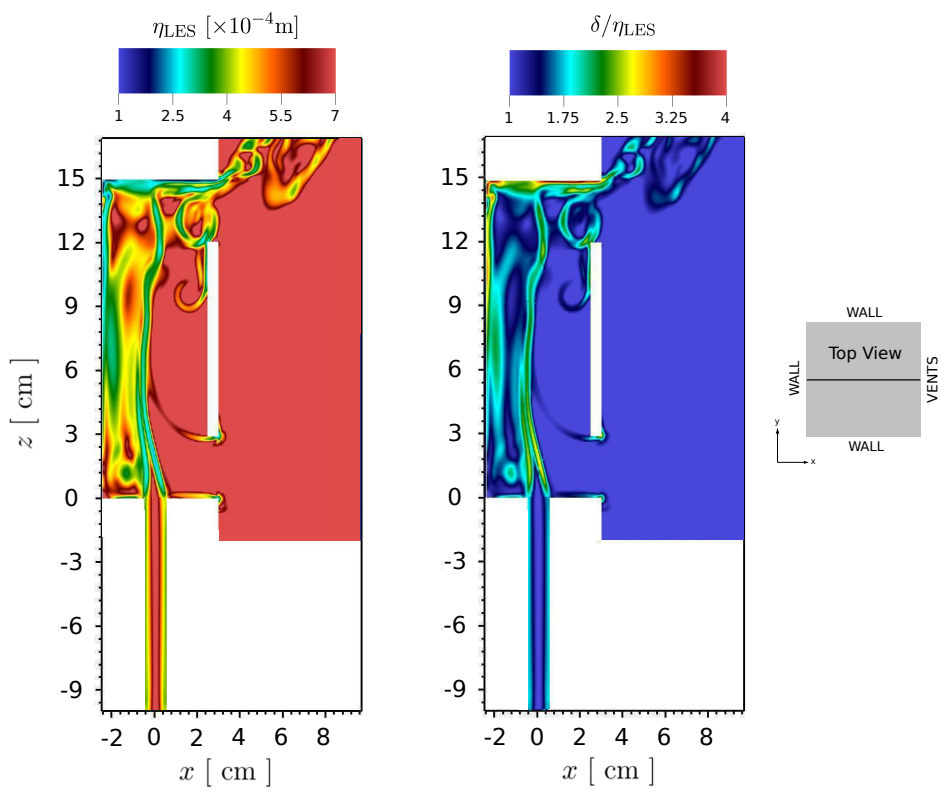

Figure 9: Instantaneous iso-contour plots at $t=106$ seconds in the mid-vertical $x z$-plane $(y=0 \mathrm{~cm})$. Left: estimated Kolmogorov length scale $\eta_{\text {LES }}$, right: ratio of the grid spacing $\delta$ to Kolmogorov length scale $\eta_{\text {LES }}$.

Three instantaneous horizontal profiles of $\delta / \eta_{\text {LES }}$ along three heights are graphed in figure 10. Largest ratios (relatively smallest Kolmogorov length scales) are situated at the jet edges (peaks on the horizontal profiles) and in the recirculation regions near the left wall facing the 
vents. On contrary, in the region near the bottom vent where the flow is almost uniform, the ratio is smaller than unity which means that the mesh is sufficiently enough to capture the small scales without requiring an eddy-viscosity model.

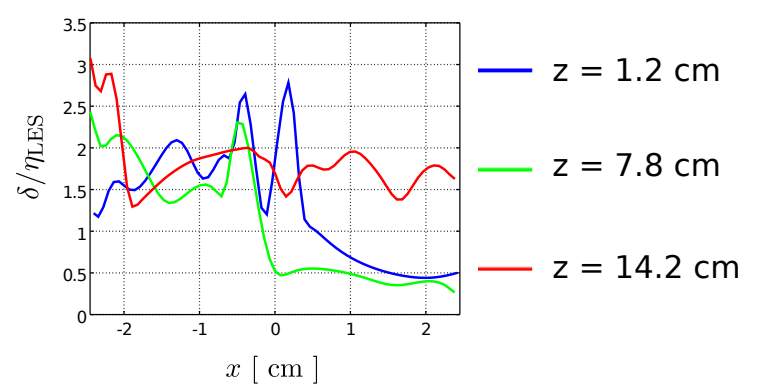

Figure 10: Horizontal and span-wise instantaneous profiles $(t=106 \mathrm{~s})$ of the grid spacing $\delta$ to Kolmogorov length scale $\eta_{\text {LES }}$ ratio. Left: vertical mid $x z$-plane $(y=0 \mathrm{~cm})$, right: vertical $y z$-plane $(x=-2 \mathrm{~cm})$.

\section{Results: influence of the exterior domain}

In the present section, the sensitivity of the flow pattern regarding the considered computational domain is presented. We keep in mind from the previous section that satisfactory convergent results independent on the extension $L x$ are obtained starting from the configuration $4_{x}$.

\subsection{Flow pattern in the cavity}

Firstly, the influence of the configuration on the flow pattern inside the cavity is illustrated by visualizing the time averaged velocity magnitude field. The iso-contours of $\langle|\mathbf{u}|\rangle_{t}$ in the mid-vertical $x z$-plane $(y=0 \mathrm{~cm})$ are depicted for all cases in figure 11.
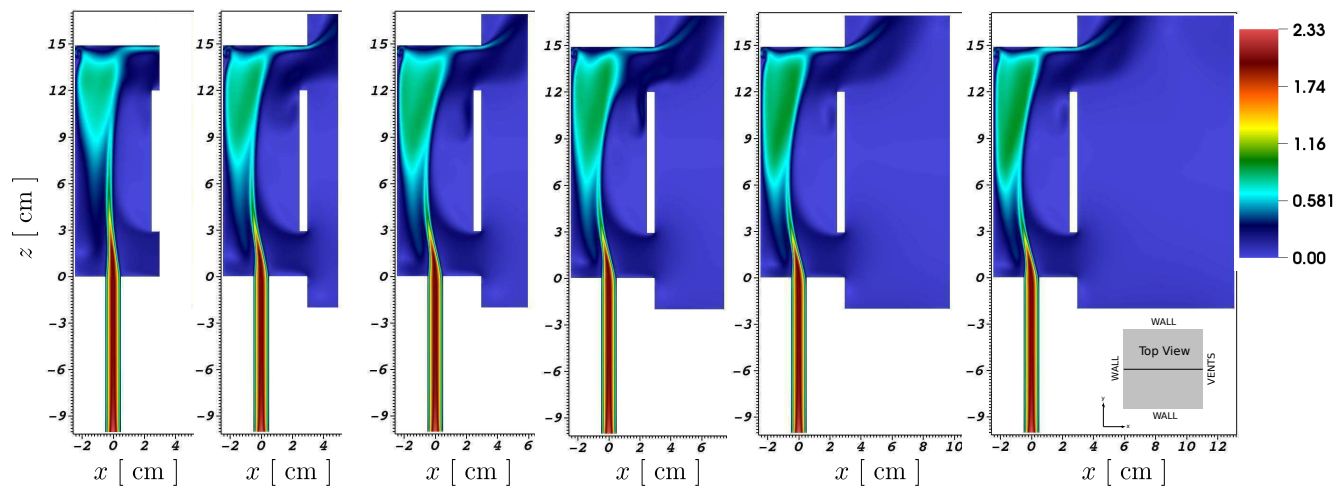

Figure 11: Time averaged flow pattern illustrated by the velocity magnitude $<|\mathbf{u}|>_{t}\left[\mathrm{~m} \cdot \mathrm{s}^{-1}\right]$ iso-contours in the mid vertical $x z$-plane $(y=0 \mathrm{~cm})$. Left to right: configurations $0_{x}$ to $5_{x}$. 


\subsubsection{Similar flow-pattern independent of the computational domain}

Let us explain the similar flow pattern observed in all cases by focussing on the distribution predicted with configuration $4_{x}$ (sufficient for convergence). Helium is injected with the highest velocity at the bottom of the pipe. When the fluid reaches the top of the pipe, it enters the cavity following a small deviation towards the left wall. This is clearly due to the ambient air that enters from the bottom vent and impacts the axis of the light jet, thus pushing the axis of the jet to the left.

Afterwards, the flow starts to spread all along the height of the cavity where we see that it occupies almost all the left part of the cavity where it remains attached with the left wall. When the buoyant jets impacts the top-ceiling, it splits into two parts where the first follows a recirculating motion at the left corner, while the second leaves the cavity from the top vent through a thin "exiting jet" situated at the top. The flow pattern near the top-ceiling is depicted in figure 12 using the time averaged velocity vector field in a zoomed region at the top of the cavity in the mid vertical $x z$-plane $(y=0 \mathrm{~cm})$. The position if the recirculating zone and the exiting jet are clearly indicated.

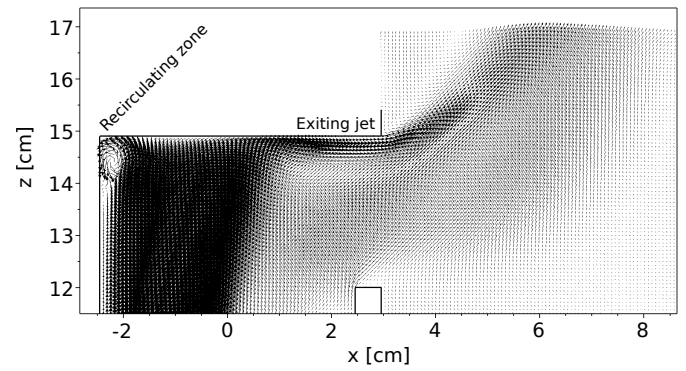

Figure 12: Time averaged flow pattern near the top ceiling illustrated by the velocity vector field in a zoomed region at the top of the mid vertical $x z$-plane $(y=0 \mathrm{~cm})$.

\subsubsection{Different flow-pattern dependent of the computational domain}

A significant influence of the computational domain is noted on the inclination angle of the buoyant jet axis (figure 11). In particular, we note that the inclination of the jet axis towards the left wall is much sharper in configurations that employ an exterior domain and that it is strengthened by increasing $L x$ (configurations $1_{x}$ to $5_{x}$ ). The issue of the slighter or sharper inclination is clearly reflected on the structure of the plume region situated in the top left part of the cavity. Although in all cases the plume is deviated from the mid height to the direction of the top vent, the buoyant zone seems to be wider in configuration $0_{x}$ with respect to the horizontal dimension. This is in contrary to the remaining configurations where the iso-contours illustrate that the plume horizontal width is reduced by increasing the size of $L x$ and thus takes a larger size in height. As expected, it can be clearly noted that almost the same qualitative distribution is reported in configurations $4_{x}$ and $5_{x}$.

Another significant influence of the computational domain can be viewed by looking to the rms field of the velocity magnitude; $\operatorname{rms}\{|\mathbf{u}|\}_{t}$. The iso-contours in the same mid vertical $x z$-plane $(y=0)$ are depicted in figure 13. In all cases, we note that $\operatorname{rms}\{|\mathbf{u}|\}_{t}$ reaches its maximum at the top of the plume, due to the impact with the top ceiling, and near the top vent due to the interaction between the interior and exterior environments (figure 13, top). 

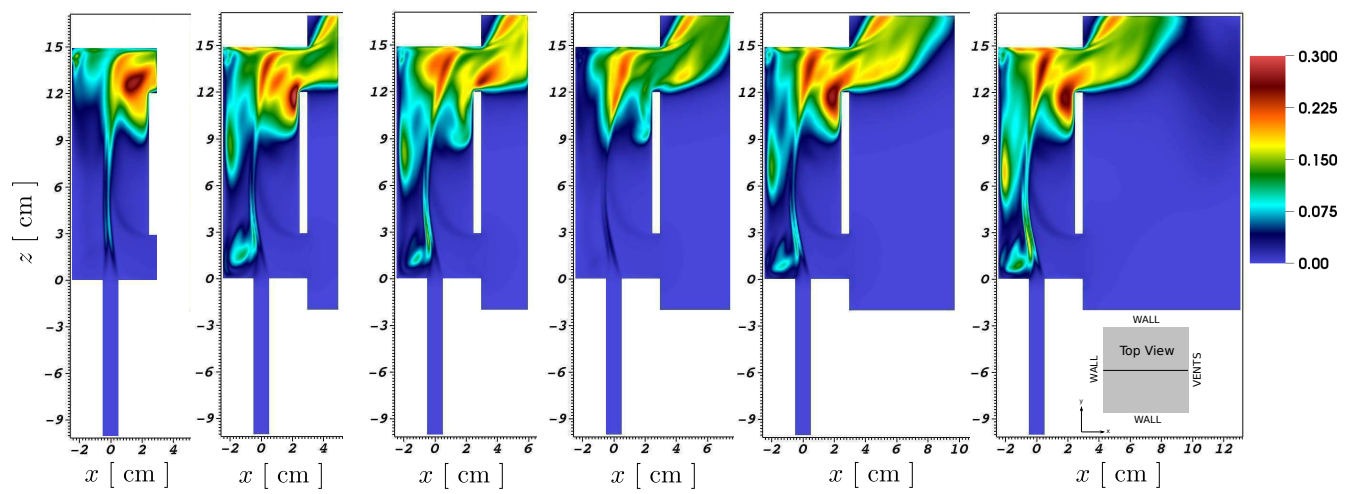

Figure 13: RMS iso-contours of the velocity magnitude $\operatorname{rms}\{|\mathbf{u}|\}_{t}\left[\mathrm{~m} \cdot \mathrm{s}^{-1}\right]$ in the mid-vertical $x z$-plane $(y=0 \mathrm{~cm})$. Left to right: configurations $0_{x}$ to $5_{x}$.

However, we can note that the rms fields are influenced by taking an exterior domain into account. In configuration $0_{x}$, oscillations are mainly located along the buoyant-jet axis, the recirculating flow at the top-left corner and near the top vent. To the contrary, additional oscillations are predicted with the configurations that take into account an exterior region (except configuration $3_{x}$ ). These additional oscillations are located in the zones near the left wall and the bottom left corner of the cavity. Excluding the configuration $3_{x}$, we note that the mean-deviations increase with the extension length $L x$.

The dependency of the oscillation levels on the configuration can be also described in figure 14 by the $\operatorname{rms}\{|\mathbf{u}|\}_{t}$ iso-contours in the vertical $y z$-plane $(x=-1.5 \mathrm{~cm})$. Without considering an exterior domain (configuration $0_{x}$ ), the rms is mainly significant at the top of the cavity where the fluid flows in two counter-rotating vortices. It can be stated here that the formation of these two vortices are predicted in all the simulations due to the continuous injection and the presence of the span-wise lateral boundaries. A time averaged distribution reproducing the top-two vortices is illustrated in figure 15 using the velocity magnitude vector field.
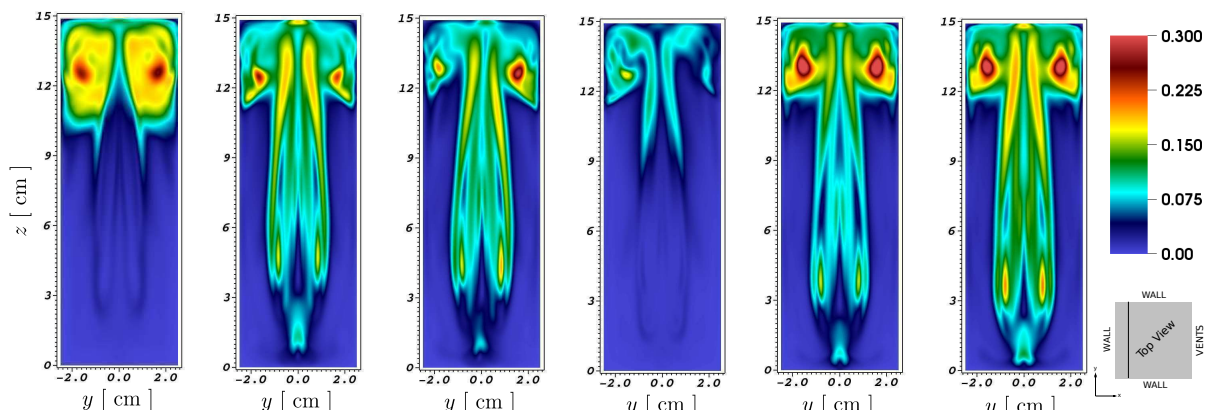

Figure 14: RMS iso-contours of the velocity magnitude $\operatorname{rms}\{|\mathbf{u}|\}_{t}\left[\mathrm{~m} \cdot \mathrm{s}^{-1}\right]$ in the vertical $y z$-plane $(x=-1.5 \mathrm{~cm})$. Left to right: configurations $0_{x}$ to $5_{x}$.

In the contrary with configuration $0_{x}$, the remaining simulations (except case $3_{x}$ ) predict additional oscillations along and near the jet axis (figure 14). However, the rms intensity increases successively as we increase $L x$. In this plane, the rms distribution is not trivially similar in the 


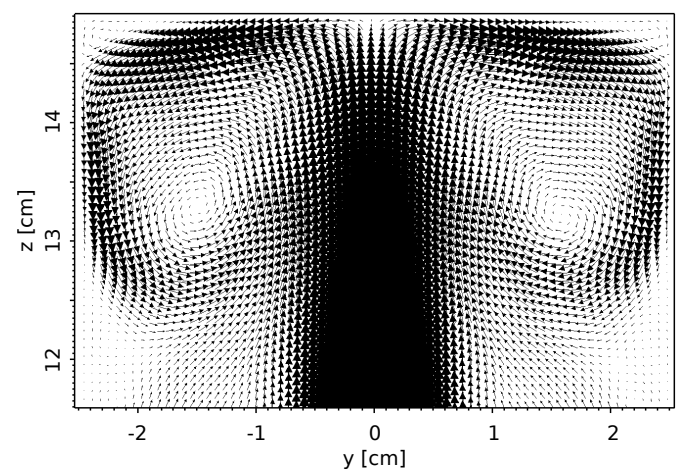

Figure 15: Time averaged vector field of the velocity magnitude $|\mathbf{u}|$ at the top of the vertical $y z$-plane $(x=-1.5 \mathrm{~cm})$ exhibiting the predicted counter-rotating vortices formed at the top of the cavity: configuration $4_{x}$.

last two cases, which is justified as far as the convergence of the deviated quantities is usually the slowest.

In conclusion, it can be stated that both the time averaged and rms quantities are different in configuration $0_{x}$ while compared with other cases. As expected, the time averaged fields show qualitative convergence in configurations $4_{x}$ and $5_{x}$, unlike the rms that probably require more convergent statistics. The reduced oscillations levels predicted with configuration $3_{x}$ require further investigations to be carried out in a future work.

\subsection{Flow pattern near the bottom vent}

Here, we consider the influence of the configuration on the flow pattern in the lower part of the cavity due to the air inflow traversing the bottom vent. In the lower part of the cavity, the flow is influenced by the presence of a computed exterior domain or not. Qualitatively speaking, the general flow pattern in the lower part of the cavity is rather similar for configurations $1_{x}$ to $5_{x}$ and thus independent of the extension $L_{x}$. Therefore, the first comparisons are presented only for configurations $0_{x}$ and $4_{x}$.

To illustrate on the different inflow behavior of the ambient air entering the cavity from the bottom vent, we consider the time averaged velocity streamlines in a zoomed region situated at the lower part of the mid-vertical $x z$-plane $(y=0 \mathrm{~cm}$, figure 16$)$.

The time averaged velocity stream-lines depict that air enters configuration $0_{x}$ in almost a uniform parallel way, unlike the other case where the inflow is not uniform. For a quantitative comparison, we consider the vertical profiles of the time averaged $\left\langle u_{1}\right\rangle_{t}$ horizontal velocity component normal to the bottom vent surface. Along the exterior surface of the bottom vent, the six vertical profiles are presented in figure 17.

An almost linear distribution is recorded along about $1 / 2$ of the vent's height in the configuration that does not take into account an exterior domain. However, the profiles for the remaining configurations are rather the same, taking almost an inverted parabola distribution with maximum absolute values situated at the extremities of the vent. We note that the profiles are slightly modified by the variation of $L_{x}$ and that they are almost superposed, as expected, in the last two configurations $\left(4_{x}\right.$ and $\left.5_{x}\right)$. 

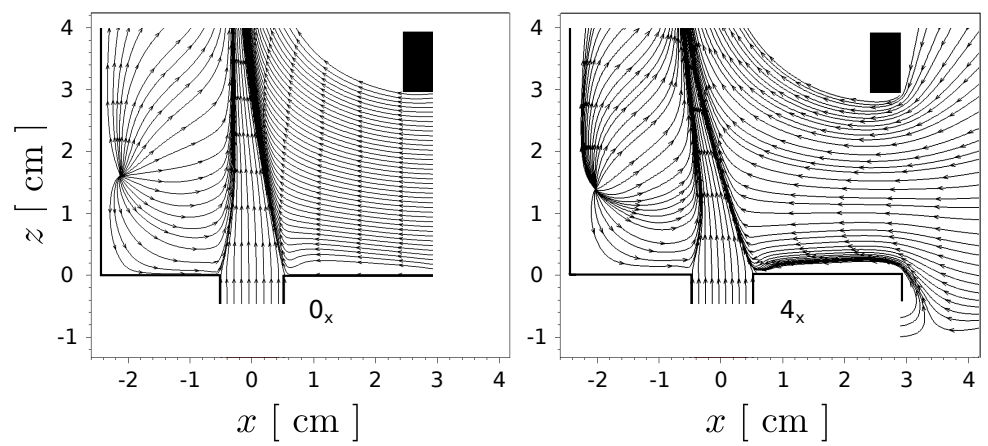

Figure 16: Time averaged flow pattern illustrated by $\langle\mathbf{u}\rangle_{t}$ stream-lines in a zoomed part of the bottom cavity situated in the mid-vertical $x z$-plane $(y=0 \mathrm{~cm})$. Left: configuration $0_{x}$, right: configuration $4_{x}$.

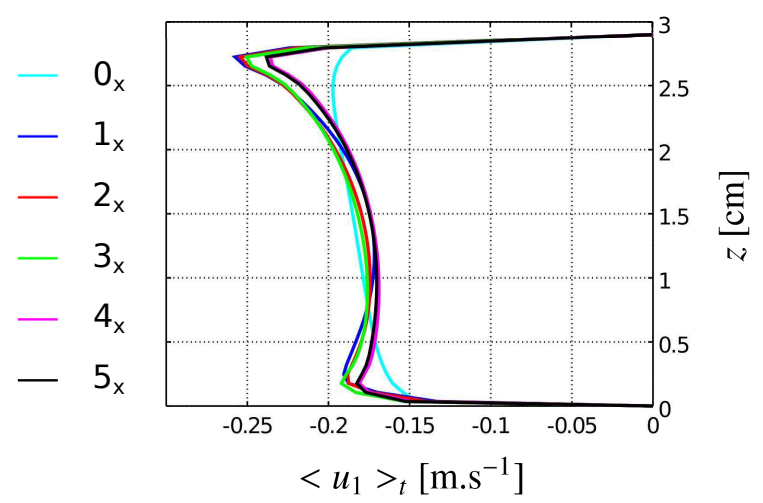

Figure 17: Time averaged $\left\langle u_{1}\right\rangle_{t}$ mid-vertical profiles $(y=0)$ in the vertical $y z$-plane $(x=2.95 \mathrm{~cm})$ at the bottom vent for configurations $0_{x}$ to $5_{x}$.

\subsection{Flow pattern near the top vent}

In this subsection, we consider the influence of the configuration in the upper part of the cavity; basically at the outer surface of the top vent $(x=2.95 \mathrm{~cm})$. The mixture outflow is illustrated for all configurations in figure 18, where the iso-contours of time averaged horizontal velocity component $\left\langle u_{1}\right\rangle_{t}$ are depicted.

In all cases, the mixture leaves the cavity with the highest velocity $<u_{1}>_{t} \approx 0.65 \mathrm{~m} . \mathrm{s}^{-1}$ from a thin layer situated at the top of the vent. However, the $\left\langle u_{1}\right\rangle_{t}$ distribution is highly influenced by the employed configuration. In configuration $0_{x}$, the distribution of $\left\langle u_{1}\right\rangle_{t}$ at the top vent surface is almost symmetrical, with a small inflow along the whole vent's width at the bottom (thick black line). This distribution is rather expected as far as we consider a completely symmetrical cavity with respect to the injection surface.

While taking into account an exterior domain, a non-symmetrical distribution is observed in particularly with configurations $2_{x}$ and $3_{x}$, to the contrary of that observed with $1_{x}, 4_{x}$ and $5_{x}$. Observing such a non-symmetrical distribution probably means that the statistical accumulations performed on configurations $2_{x}$ and $3_{x}$ have not been carried out on a pure quasi-steady solution 

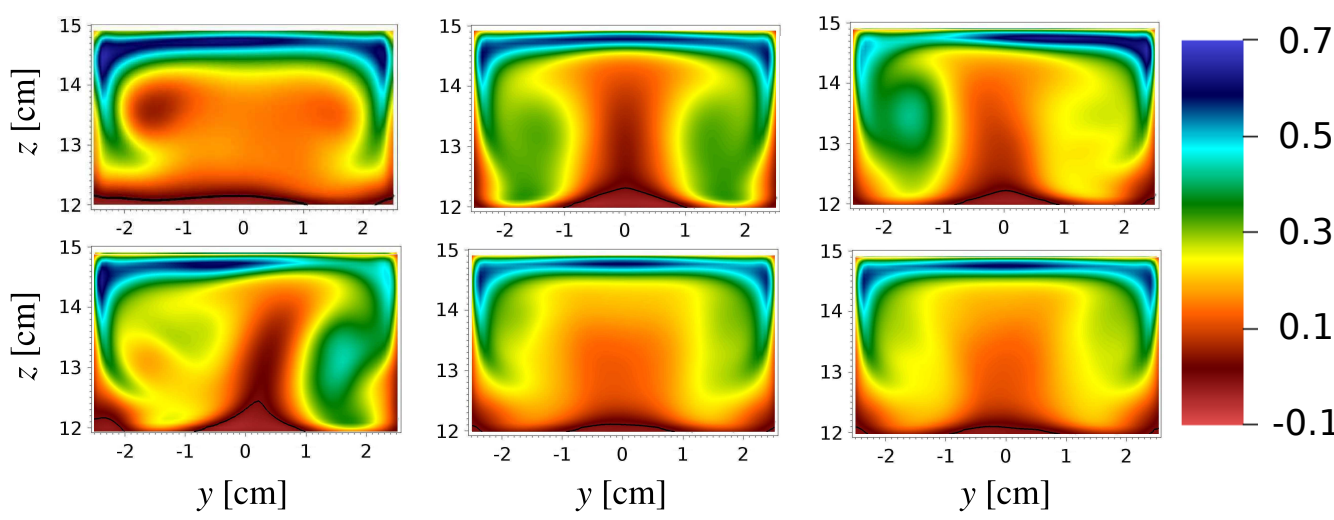

Figure 18: Time averaged flow pattern in the vertical $y z$-plane $(x=2.95 \mathrm{~cm})$ at the top vent exterior surface: $\left\langle u_{1}>\right.$ $\left[\mathrm{m} . \mathrm{s}^{-1}\right] x$-horizontal velocity component iso-contours. Thick black lines of the contour plots denotes the zero contour line indicating the limit of the back flow. Left to right, top to bottom: configurations $0_{x}$ to $5_{x}$.

and that a longer time is required to finish the transient solution. However, we note again that a small inflow takes place at the bottom of the vent in all case.

A quantitative comparison regarding the influence of the exterior region is illustrated in figure 19 by the considering the mid-vertical profiles of $\left\langle u_{1}\right\rangle_{t}(y=0$ in the same plane). Almost all profiles are confounded in the thin layer at the upper part of the top vent where the velocity of the exiting jet is reproduced. Here, the maximum horizontal velocity value is predicted, although the value in configuration $0_{x}$ is greater by about $4 \%$ compared to configurations $1_{x}$ to $5_{x}$.

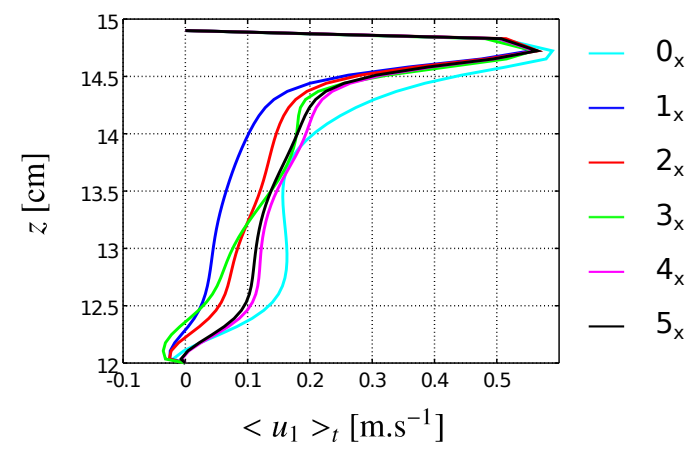

Figure 19: Time averaged $\left\langle u_{1}\right\rangle_{t}$ mid-vertical profiles $(y=0)$ in the vertical $y z$-plane $(x=2.95 \mathrm{~cm})$ at the top vent for configurations $0_{x}$ to $5_{x}$.

In the lower part of the top vent, more dispersed profiles are seen. Regarding configurations $4_{x}$ and $5_{x}$, the distribution is rather similar with slight discrepancies where we note almost superposed profiles.

\section{Helium distribution and a preliminary comparison versus the Linden's theoretical model}

In this section, we describe the helium distribution in the cavity by analyzing the fields obtained from the computational domain $4_{x}$. The helium volume fraction $X_{1}$ is defined as 
$X_{1}=\left(\rho-\rho_{\mathrm{amb}}\right) /\left(\rho_{\mathrm{inj}}-\rho_{\mathrm{amb}}\right)=\rho Y_{1} / \rho_{\mathrm{inj}}$

\subsection{Helium oscillations and stratification}

In figure 20, we depict the rms iso-contours of the helium mass fraction $\operatorname{rms}\left\{Y_{1}\right\}_{t}$ (left) and the time averaged line contours of the helium volume fraction $\left\langle X_{1}\right\rangle_{t}$ (left) and in the mid-vertical $x z$-plane $(y=0 \mathrm{~cm})$.

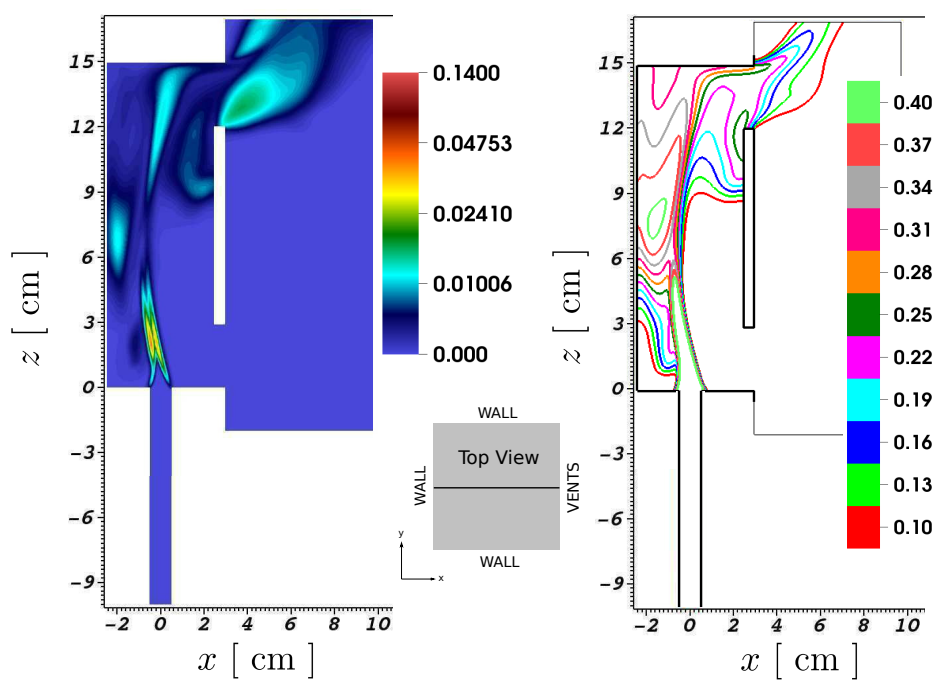

Figure 20: Configuration $4_{x}$, mid-vertical $x z$-plane $(y=0 \mathrm{~cm})$. Left: rms iso-contours of the helium mass fraction $\operatorname{rms}\left\{Y_{1}\right\}_{t}$, right: line contours of the time averaged helium volume fraction $\left.<X_{1}\right\rangle_{t}$.

Unlike the distribution of $\operatorname{rms}\{|\mathbf{u}|\}_{t}$ which indicates that the maximum velocity oscillations take place at the top of the cavity (recall figures 13 and 14), the maximum $\operatorname{rms}\left\{Y_{1}\right\}_{t}$ is located in the lower part of the cavity; basically at the jet edges. This is rather logical and is due to the significant concentration gradients caused by the heavy air impacting the rising light fluid at this position (figure 20, left). Although of smaller intensity, $Y_{1}$ oscillations are also recorded near the left wall, in the plume (upper part of the buoyant jet), near the top ceiling due to the impact and in the vicinity of the top vent due to the recirculating regions.

Regarding the helium distribution (figure 20, right), helium stratification layers are predicted to form at the left and right regions of the cavity. The layers at the left descend more than those at the right, which is justified by the low pressure region in the vicinity of the lower-left corner (lower layers) and the mixture that is exiting continuously from the top vent (higher layers).

Similarly, stratified helium layers are formed in the span-wise directions parallel to the vents surfaces. However, the distribution in these directions is rather symmetrical, as expected. We illustrate for example in figure 21 the symmetrical stratification that takes place in the vertical $y z$-plane situated at a horizontal position $x=-1.5 \mathrm{~cm}$.

\subsection{LES predictions versus Linden's pre-safety calculation model}

We present in here a preliminary comparison of the helium concentration distribution versus the theoretical model of Linden et al. [8]. This model is generally used in hydrogen safety precalculations of two vented idealized fuel cell configurations [26], similar to the geometry studied 


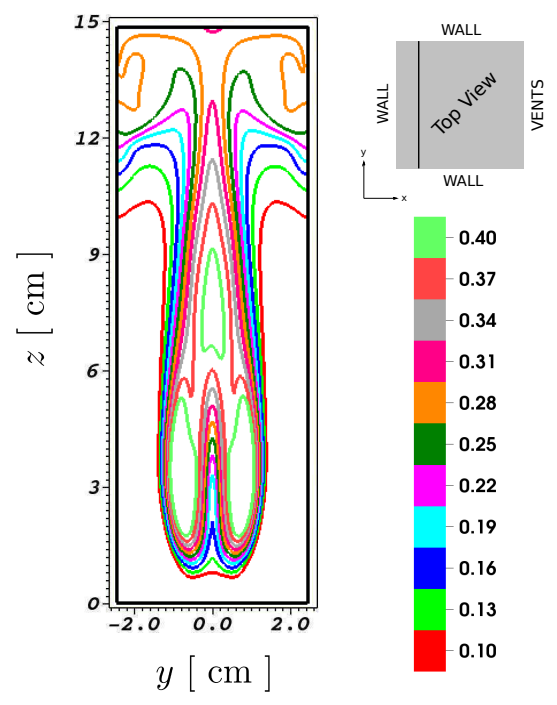

Figure 21: Line contours of the time averaged helium volume fraction $<X_{1}>_{t}$ in the vertical $y z$-plane $(x=-1.5 \mathrm{~cm})$ : configuration $4 x$

in this paper. In situations where a bi-layer distribution takes place inside the two vented cavity, the model can be correctly served to predict the height of the separating interface, in addition to the homogeneous concentration at the top. A summary of the concerned model can be reviewed from [3].

Clearly from the distribution of $\left\langle X_{1}\right\rangle_{t}$ (figures 20 and 21), the theoretical model of Linden et al. [8] can not be correctly applied as far as stratified layers are formed. However, we compare in what follows the numerical distribution with the theory to see if the model prediction, regarding the maximum concentration in the cavity (away from the buoyant jet axis), is coherent with the numerical calculation.

The LES $<X_{1}>_{t}$ distribution inside the cavity can be described by three vertical profiles: along the inclined buoyant jet axis, near the middle of the left wall where the jet deviates towards it, and finally, by the mean concentration distribution in the far field away from the buoyant jet axis. The three vertical profiles are plotted versus the height of the cavity in figure 22. Along the buoyant jet axis (solid black line) and near the middle of the left wall (dashed black line), the profiles seems to be almost identical starting from about a $z=8 \mathrm{~cm}$. This observation is rather logical since at this position, the buoyant jet is mainly situated near the left wall (due to the deviation of the axis). It can also be noted that the stratification near the buoyant jet axis (black lines) is weaker than that in the far fields (blue line), where in the last case the stratification is almost linear.

We plot on the same figure 22 (dashed red line) the bi-concentration profile estimated from the model of Linden et al. [8]. The theoretical calculation predict that the lower interface of the homogeneous layer, which constitutes approximately of $30-31 \%$ of helium, is situated a bout a height $z \approx 7.5 \mathrm{~cm}$. Clearly, the simulated configuration is not situated in the framework of this theory, although the numerical profiles show somehow a virtual plateau reproducing a homogeneous layer formation at the top of the cavity; above $12 \mathrm{~cm}$ in the far fields (blue line) and above $9 \mathrm{~cm}$ near the buoyant jet (black profiles). However, the maximum LES concentration 


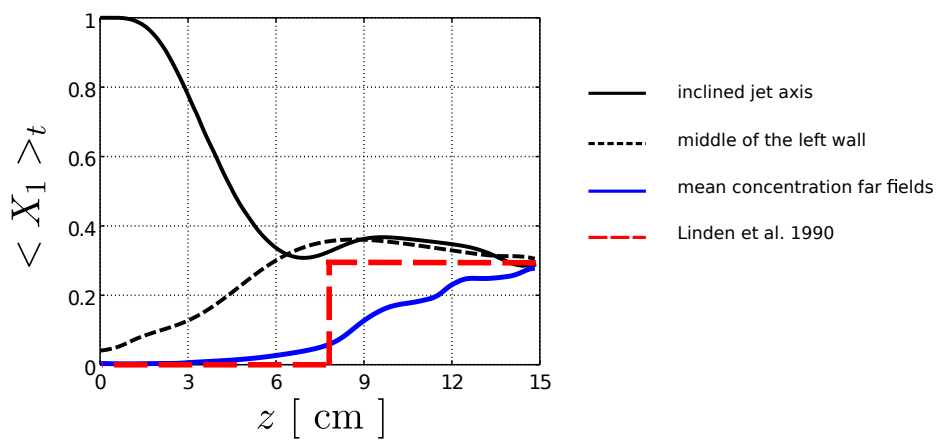

Figure 22: Time averaged helium volume fraction $\left\langle X_{1}\right\rangle_{t}$ vertical profiles along the inclined buoyant jet axis (solid black line) and near the middle of the left wall (dashed black line). The far field concentrations are represented by the mean $\left\langle X_{1}\right\rangle_{t}$ vertical profile (solid blue line). The dashed red line describes the distribution predicted by the theory of Linden et al. [8].

at the top of the cavity is in good agreement with the theoretical prediction.

Being far from the Linden's configuration can be justified by the main reason that the bilayer distribution is not valid. In particular, the highly confined flow simulated in this paper induces direct interactions between the buoyant jet and both, the walls and exterior environment, which thus opposes the formation of a 1D bi-layer concentration distribution. However, the fact that maximum concentration is in good agreement with the theoretical prediction is due to the satisfactory LES prediction of the air-inflow traversing the bottom vent. This agreement can be reviewed from the LES-PIV comparisons presented in [4]. In other words, since the ratio between that air inflow flux (bottom vent) and the injected helium flux is directly connected to that maximum concentration in the cavity, the agreement of the maximum concentration is justified.

\section{Discussions and concluding remarks}

A binary mixture buoyant jet developing in a cavity with two vents has been modelled numerically by LES. The influence of considering an exterior domain in the calculations is carried out on six numerical simulations with the CEA TRUST-TrioCFD open source code. It has been illustrated that a small role is played by the sub-grid scale model and that the performed LES is well resolved. From a convergence study, a sufficient computational domain has been identified so that the flow inside the vented cavity becomes independent of the exterior environment.

In the different simulations, a qualitative similar flow pattern has been predicted; basically the regarding the velocity distribution, in addition to the deformation and the inclination of the buoyant jet axis. However, discrepancies have been noted on the global averaged quantities; like the volumetric and mass flow-rates. For example, without modelling an exterior domain, the volumetric flow rate of air inflow is underestimated when compared to the other cases, and thus the total mass of helium is overestimated inside the cavity. Regarding the concentration distribution inside the cavity, it has been figured out that a stratified regime builds inside the cavity. Hence, the simulated configuration is not situated in the framework of the classical twovented idealized fuel cells configurations, where a bi-layer distribution is usually seen, and thus the theoretical model of Linden et al. [8] is not suitable for this study. 
On the one hand, for the preliminary hydrogen safety pre calculations, we can recommend at present an LES on a simple configuration without an exterior domain. It is cheap in computational cost, conservative and somehow acceptably predicts the general flow pattern. It is true that the mass is overestimated compared with other situations, but it can be stated that overestimating the concentration rather than underestimating it is recommended in safety studies. On the other hand, defining the correct boundary conditions that can be imposed directly on the vents without modelling an exterior region remain as a main future perspective. If this point is achieved, the computational cost will be reduced and satisfactory predictions can be presented only by modelling the cavity.

Finally, it will be very interesting also to identify the design parameters that limits the formation of a bi-layer concentration regime. This point is extremely important for hydrogen safety assessments as far as it can serve in improving the theoretical models that might be cheaper to use, comparing with the CFD approaches.

\section{Acknowledgements}

The authors would like to gratefully thank Yann Fraigneau, Ulrich Bieder, Gautier Fauchet and the CEA TRUST-Trio CFD support team for the interesting discussions and support. Special thanks for the project GENCI 2A0326 that allowed us to access also the super calculators at IDRIS.

\section{References}

[1] C. J. Chen, W. Rodi, Vertical turbulent buoyant jets: a review of experimental data, NASA STI/Recon Technical Report A 80.

[2] W. Rodi, Turbulent buoyant jets and plumes, Vol. 3, Pergamon press Oxford, 1982.

[3] G. Bernard-Michel, E. Saikali, D. Houssin, Experimental measurements, cfd simulations and model for a helium release in a two vents enclosure, in: Proceeding of the International Conference on Hydrogen Safety, Hamburg, Germany, 2017.

[4] E. Saikali, Numerical modelling of an air-helium buoyant jet in a two vented enclosure, Ph.D. thesis, Sorbonne Université (2018).

[5] G. Bernard-Michel, D. Houssin-Agbomson, Comparison of helium and hydrogen releases in $1 \mathrm{~m} 3$ and $2 \mathrm{~m} 3$ two vents enclosures: Concentration measurements at different flow rates and for two diameters of injection nozzle, International Journal of Hydrogen Energy 42 (11) (2017) 7542-7550.

[6] G. Blanquart, H. Pitsch, Large-eddy simulation of a turbulent buoyant helium plume, Bulletin of the American Physical Society 53.

[7] G. Desrayaud, E. Chénier, A. Joulin, A. Bastide, B. Brangeon, J. Caltagirone, Y. Cherif, R. Eymard, C. Garnier, S. Giroux-Julien, et al., Benchmark solutions for natural convection flows in vertical channels submitted to different open boundary conditions, International Journal of Thermal Sciences 72 (2013) 18-33.

[8] P. Linden, G. Lane-Serff, D. Smeed, Emptying filling boxes: the fluid mechanics of natural ventilation, Journal of Fluid Mechanics 212 (1990) 309-335.

[9] R. Kalter, M. J. Tummers, J. B. W. Bettink, B. W. Righolt, S. Kenjereš, C. R. Kleijn, Aspect ratio effects on fluid flow fluctuations in rectangular cavities, Metallurgical and Materials Transactions B 45 (6) (2014) 2186-2193.

[10] F. Williams, Combustion Theory, Addison-Wesley series in engineering sciences, Addison-Wesley Publishing Company, 1965.

URL https://books.google.fr/books?id=JzxRAAAAMAAJ

[11] D. D. Gray, A. Giorgini, The validity of the boussinesq approximation for liquids and gases, International Journal of Heat and Mass Transfer 19 (5) (1976) 545 - 551. doi:https://doi.org/10.1016/0017-9310(76)90168-X.

[12] B. Müller, Low mach number asymptotics of the navier-stokes equations and numerical implications (1999).

[13] C. Wilke, A viscosity equation for gas mixtures, The journal of chemical physics 18 (4) (1950) 517-519.

[14] J. Smagorinsky, General circulation experiments with the primitive equations: I. the basic experiment, Monthly weather review 91 (3) (1963) 99-164. 
[15] DEN-CEA, TRUST-TrioCFD code version 1.7.4, http://www-trio-u.cea.fr.

[16] E. Saikali, A. Sergent, G. Bernard-Michel, C. Tenaud, Large eddy simulations of an air-helium buoyant jet in a two vented enclosure: influence of the outlet boundary condition, in: Proceeding of the 23ème Congrès Français de Mécanique, Lille, France, 2017.

[17] E. Saikali, G. Bernard-Michel, A. Sergent, C. Tenaud, R. Salem, Highly resolve large eddy simulations of a transitional air-helium buoyant jet in a two vented enclosure: validation against particle image velocimetry experiments, in: Proceeding of the International Conference on Hydrogen Safety, Hamburg, Germany, 2017.

[18] A. Wambecq, Rational runge-kutta methods for solving systems of ordinary differential equations, Computing 20 (4) (1978) 333-342.

[19] B. Leonard, A stable and accurate convective modelling procedure based on quadratic upstream interpolation, Computer Methods in Applied Mechanics and Engineering 19 (1) (1979) 59 - 98. doi:http://dx.doi.org/10.1016/00457825(79)90034-3.

[20] Y. Saad, Iterative methods for sparse linear systems, SIAM, 2003.

[21] J.-L. Guermond, P. Minev, J. Shen, An overview of projection methods for incompressible flows, Computer methods in applied mechanics and engineering 195 (44) (2006) 6011-6045.

[22] S. Chhabra, P. Huq, A. K. Prasad, Characteristics of small vortices in a turbulent axisymmetric jet, Journal of fluids engineering 128 (3) (2006) 439-445.

[23] S. Pope, Turbulent Flows, Cambridge University Press, 2000. URL https://books . google.fr/books?id=HZsTw9SMx-OC

[24] G. Maragkos, P. Rauwoens, Y. Wang, B. Merci, Large eddy simulations of the flow in the near-field region of a turbulent buoyant helium plume, Flow, Turbulence and Combustion 90 (3) (2013) 511-543. doi:10.1007/s10494012-9437-5.

URL http://dx.doi.org/10.1007/s10494-012-9437-5

[25] M. V. Salvetti, B. Geurts, J. Meyers, P. Sagaut, Quality and Reliability of Large-Eddy Simulations II, Vol. 16, Springer Science \& Business Media, 2010.

[26] B. Fuster, D. Houssin-Agbomson, S. Jallais, E. Vyazmina, G. Dang-Nhu, G. Bernard-Michel, M. Kuznetsov, V. Molkov, B. Chernyavskiy, V. Shentsov, et al., Guidelines and recommendations for indoor use of fuel cells and hydrogen systems, International Journal of Hydrogen Energy 42 (11) (2017) 7600-7607. 


\section{Nomenclature}

\begin{tabular}{|c|c|c|c|c|c|}
\hline $\mathrm{ACF}$ & Auto correlation function & & DNS & Direct numerical simulations & \\
\hline LES & Large eddy simulations & & PIV & Particle image velocimetry & \\
\hline SGS & Sub-grid scale & & $a m b$ & Subscript denoting ambient & \\
\hline inj & Subscript denoting injection & & $\mathrm{rms}$ & Root mean square & \\
\hline & Space filter symbol & & $\sim$ & Favre averaging symbol & \\
\hline$<\cdot>_{t}$ & Time averaging operator & & & & \\
\hline$C_{s}$ & Smagorinsky coefficient & {$[-]$} & $D$ & Mixture diffusion coefficient & {$\left[\mathrm{m}^{2} \cdot \mathrm{s}^{-1}\right]$} \\
\hline$E_{k}$ & Kinetic energy & {$\left[\mathrm{m}^{2} \cdot \mathrm{s}^{-2}\right]$} & $H$ & Height of cavity & {$[\mathrm{m}]$} \\
\hline$L$ & Span-wise length of cavity & {$[\mathrm{m}]$} & $M$ & Molar mass & {$\left[\mathrm{Kg} \cdot \mathrm{mol}^{-1}\right]$} \\
\hline Мa & Mach number & {$[-]$} & $P$ & Hydrodynamic pressure & {$[\mathrm{Pa}]$} \\
\hline$Q$ & Volumetric flow-rate & {$\left[\mathrm{m}^{3} \cdot \mathrm{s}^{-1}\right]$} & $R$ & Specific gas constant & {$\left[\mathrm{J} . \mathrm{K}^{-1} \cdot \mathrm{mol}^{-1}\right]$} \\
\hline $\operatorname{Re}$ & Reynolds number & {$[-]$} & $\mathrm{Ri}$ & Richardson number & {$[-]$} \\
\hline$S c$ & Schmidt number & {$[-]$} & $T$ & Temperature & {$[\mathrm{K}]$} \\
\hline$W$ & Horizontal width of cavity & {$[\mathrm{m}]$} & $X_{1}$ & Helium volume fraction & {$[-]$} \\
\hline$Y_{1}$ & Helium mass fraction & {$[-]$} & $Y_{2}$ & Air mass fraction & {$[-]$} \\
\hline $\mathbf{u}$ & Mixture mass velocity vector & {$\left[\mathrm{m} \cdot \mathrm{s}^{-1}\right]$} & $\mathbf{x}$ & Space vector & {$[\mathrm{m}]$} \\
\hline$d$ & Diameter of pipe & {$[\mathrm{m}]$} & $f$ & Frequency & {$[\mathrm{Hz}]$} \\
\hline$g$ & Gravity vector & {$\left[\mathrm{m} . \mathrm{s}^{-2}\right]$} & $h$ & Height of pipe & {$[\mathrm{m}]$} \\
\hline$p$ & Thermodynamic pressure & {$[\mathrm{Pa}]$} & $q$ & Mass flow-rate & {$\left[\mathrm{Kg} . \mathrm{s}^{-1}\right]$} \\
\hline$t$ & Time & {$[\mathrm{s}]$} & & & \\
\hline $\mathcal{M}_{\mathrm{He}}$ & Helium mass in the cavity & {$[\mathrm{Kg}]$} & $O$ & Cartesian system's origin & {$[-]$} \\
\hline$\Delta$ & Filter width & {$[\mathrm{m}]$} & $\delta$ & Mesh step size & {$[\mathrm{m}]$} \\
\hline$\delta t$ & Time step & {$[\mathrm{s}]$} & $\epsilon$ & Total dissipation rate & {$\left[\mathrm{m}^{2} \cdot \mathrm{s}^{-3}\right]$} \\
\hline$\eta$ & Kolmogorov length scale & {$[\mathrm{m}]$} & $\lambda_{f}$ & Taylor micro-scale & {$[\mathrm{s}]$} \\
\hline$\mu$ & Dynamic viscosity & {$\left[\mathrm{Kg} \cdot \mathrm{m}^{-1} \cdot \mathrm{s}^{-1}\right]$} & $\rho$ & Density & {$\left[\mathrm{Kg} \cdot \mathrm{m}^{-3}\right]$} \\
\hline$\tau$ & Viscous stress tensor & {$[\mathrm{Pa}]$} & & & \\
\hline
\end{tabular}

